\title{
MONASHUniversity
}

Australia

Department of Econometrics and Business Statistics

http://www.buseco.monash.edu.au/depts/ebs/pubs/wpapers/

\section{Estimation in threshold autoregressive models with a stationary and a unit root regime}

\author{
Jiti Gao Dag Tjøstheim and Jiying Yin
}

September 2011

Working Paper 21/11 


\title{
Estimation in threshold autoregressive models with a station- ary and a unit root regime
}

\author{
Jiti Gao ${ }^{\star *}$, Dag Tjøstheim ${ }^{\dagger}$ and Jiying Yin** \\ ${ }^{\star}$ School of Economics, University of Adelaide, Adelaide SA 5005, Australia \\ ${ }^{*}$ Department of Econometrics and Business Statistics, Monash University, Caulfield \\ East VIC 3145, Australia \\ ${ }^{\dagger}$ Department of Mathematics, University of Bergen, Bergen 5008, Norway
}

\begin{abstract}
This paper treats estimation in a class of new nonlinear threshold autoregressive models with both a stationary and a unit root regime. Existing literature on nonstationary threshold models have basically focused on models where the nonstationarity can be removed by differencing and/or where the threshold variable is stationary. This is not the case for the process we consider, and nonstandard estimation problems are the result.

This paper proposes a parameter estimation method for such nonlinear threshold autoregressive models using the theory of null recurrent Markov chains. Under certain assumptions, we show that the ordinary least squares (OLS) estimators of the parameters involved are asymptotically consistent. Furthermore, it can be shown that the OLS estimator of the coefficient parameter involved in the stationary regime can still be asymptotically normal while the OLS estimator of the coefficient parameter involved in the nonstationary regime has a nonstandard asymptotic distribution. In the limit, the rate of convergence in the stationary regime is asymptotically proportional to $n^{-\frac{1}{4}}$, whereas it is $n^{-1}$ in the nonstationary regime. The proposed theory and estimation method are illustrated by both simulated data and a real data example.
\end{abstract}

Key words: Autoregressive process; null-recurrent process; semiparametric model; threshold time series; unit root structure.

JEL Classification: C14, C18, C22. 


\section{Introduction}

Ordinary unit root models have just one regime, whereas ordinary threshold models have several regimes, but are stationary. In this paper, we study a threshold model that has unit-root behavior in one regime and acts as a stationary process in another regime. More specifically, we consider a parametric threshold autoregressive (TAR) model of the form

$$
y_{t}=\alpha_{1} y_{t-1} I\left[y_{t-1} \in C_{\tau}\right]+\alpha_{2} y_{t-1} I\left[y_{t-1} \in D_{\tau}\right]+e_{t}, 1 \leq t \leq n
$$

where $C_{\tau}$ is a subset of $R^{1}=(-\infty, \infty)$ indexed by $\tau>0, D_{\tau}=C_{\tau}^{c}=R^{1}-C_{\tau}$ is the complement of $C_{\tau}, \tau$ is essentially assumed to be known in the asymptotic analysis in this paper, $-\infty<\alpha_{1}, \alpha_{2}<\infty$ are assumed to be unknown parameters, but will be estimated under the assumption that $\alpha_{2}=1$, the distribution of $\left\{e_{t}\right\}$ is absolutely continuous with respect to Lebesgue measure with $p_{e}(\cdot)$ being the density function satisfying $\inf _{x \in C} p_{e}(x)>0$ for all compact sets $C,\left\{e_{t}\right\}$ is assumed to be a sequence of independent and identically distributed (i.i.d.) random errors with $E\left[e_{1}\right]=0,0<\sigma^{2}=$ $E\left[e_{1}^{2}\right]<\infty$ and $E\left[e_{1}^{4}\right]<\infty,\left\{e_{t}\right\}$ and $\left\{y_{s}\right\}$ are assumed to be mutually independent for all $s<t$, and $n$ is the sample size of the time series. Let $y_{0}=0$ throughout this paper. Even though (1.1) is the simplest possible of the type of models we are discussing, it requires nonstandard techniques using the theory of null recurrent Markov chains. A few results of this theory are reviewed in Appendix A.

The vast majority of threshold models used have been stationary models, i.e., models for which $\left|\alpha_{1}\right|<1$ and $\left|\alpha_{2}\right|<1$ in the first order case. Such models were introduced by Tong and Lim (1980). See also Tong (1983, 1990). Among later contributions, Chan $(1990,1993)$ consider both estimation and testing problems for the case where $\left\{y_{t}\right\}$ of (1.1) is stationary. His work is extended in Li and Ling (2011). Pham, Chan and Tong (1991) consider a nonlinear unit-root problem and establish strong consistency results for the ordinary least squares (OLS) estimators of $\alpha_{1}$ and $\alpha_{2}$ for the case where $\left(\alpha_{1}, \alpha_{2}\right)$ lie on the boundary, Hansen (1996) rigorously establishes an asymptotic theory for the likelihood ratio test for a threshold, Chan and Tsay (1998) discuss a related continuous-time TAR model, and Hansen (2000) proposes a new approach to 
estimating stationary TAR models. More recently, Liu, Ling and Shao (2009) extend the discussion of Pham, Chan and Tong (1991) by establishing an asymptotic distribution of the OLS estimator of $\alpha_{2}$ for the case where $C_{\tau}=(-\infty, \tau]$ and either $\alpha_{2}=1$ and $\alpha_{1}<1$ or $\alpha_{2}>1$ and $\alpha_{1} \leq 1$ hold.

There have been other extensions to the nonstationary case, see in particular Caner and Hansen (2001), thus having a class of models that allow for both nonlinearity and nonstationarity, and where these properties can be (Caner and Hansen 2001) separately tested for. The nonstationarity of these models under the null hypothesis has been of a rather restricted form, thus typically regarding both $y_{t}-y_{t-1}$ and the threshold variable to be stationary. In the first order case, this leads to a somewhat degenerate model that under the null hypothesis has $H_{0}: \alpha_{1}=\alpha_{2}=1$ in

$$
y_{t}-y_{t-1}=\left(\alpha_{1}-1\right) y_{t-1} I\left[z_{t} \in C_{\tau}\right]+\left(\alpha_{2}-1\right) y_{t-1} I\left[z_{t} \in D_{\tau}\right]+e_{t},
$$

where $\left\{z_{t}\right\}$ is a sequence of stationary threshold variables, $C_{\tau}=(-\infty, \tau]$ and $D_{\tau}=$ $(\tau, \infty)$. The parameters $\alpha_{1}$ and $\alpha_{2}$ can then be estimated under $H_{0}$, which leads to a pure random walk model for (1.2) but more general difference type models for the higher order case are treated in Caner and Hansen (2001). The authors also point out that there are several nonstationary alternatives when $H_{0}$ does not hold. One of the alternatives to $H_{0}$ is as follows:

$$
H_{1}:\left|\alpha_{1}\right| \neq 1 \text { and } \alpha_{2}=1
$$

which does not imply $y_{t}-y_{t-1}$ is stationary under $H_{1}$. For more references, including econometric interpretations of threshold effects, we refer to Teräsvirta, Tjøstheim and Granger (2010); see in particular Sections 3.2.2, 8.2.3, 11.5 and 11.8.

We allow for more general forms of nonstationarity in which we do not require $y_{t}-y_{t-1}$ to be stationary, nor do we require the threshold variable to be stationary. To the best of our knowledge, estimation in this situation has not been treated before in the literature. In the present paper, for simplicity, we only treat the first order case, but the theory can be extended to higher order and vector models, making it possible to introduce threshold cointegration models in this context. It is also possible to allow 
nonlinear behavior in the regime $C_{\tau}$. This is done by replacing the linear function $\alpha_{1} y$ by a nonparametric function, also implicitly including an intercept in the model.

Although our focus in this paper is to estimate $\alpha_{1}$ and $\alpha_{2}$ and then study asymptotic properties of the proposed estimates in Section 2.1 when $\tau$ is assumed to be known, we propose an estimation procedure for the $\tau$ parameter in Section 2.2 when $\tau$ is unknown. Since the case of both $\left|\alpha_{1}\right|<1$ and $\left|\alpha_{2}\right|<1$ and the case of $\alpha_{1}=\alpha_{2}=1$ have already been discussed in the literature (Chan 1993; Hansen 2000), we are interested in proposing an estimation method to deal with model (1.1) where $C_{\tau}$ is either a compact subset of $R^{1}$ or a set of type $(-\infty, \tau]$ or $[\tau, \infty)$, and where $\alpha_{2}=1$ and $\alpha_{1}$ may be larger or smaller than one in absolute value. Model (1.1) may be used to detect and then estimate structural change from one regime to another. Note that $\tau$ can be a vector of unknown parameters. In the case where $C_{\tau}=\left[\tau_{1}, \tau_{2}\right]$ with $-\infty<\tau_{1}<\tau_{2}<\infty$, $\tau=\left(\tau_{1}, \tau_{2}\right)$. It is shown in Section 2 that the OLS estimator of $\alpha_{1}$ is asymptotically consistent with a rate of convergence which in the limit is proportional to $n^{-\frac{1}{4}}$ where we can even let $\left|\alpha_{1}\right|>1$ when $C_{\tau}$ is compact. By contrast, the OLS estimator of $\alpha_{2}$ is asymptotically consistent with the super $n$-rate of convergence. In a related paper by Liu, Ling and Shao (2009), the authors have established similar results for $\widehat{\alpha}_{2}$, but have not established any asymptotic theory for $\widehat{\alpha}_{1}$.

The organization of this paper is as follows. Section 2 establishes asymptotic distributions of the OLS estimators of $\alpha_{1}$ and $\alpha_{2}$ and contains an estimation procedure for the threshold parameter $\tau$. Section 3 discusses an extension of model (1.1) to a semiparametric threshold autoregressive (SEMI-TAR) model. Examples of implementation are given in Section 4. The paper concludes in Section 5. We will use the theory of $\beta$-null recurrent Markov chains in this paper and some general results about these processes are given in Appendix A. Much more details can be found in Karlsen and Tjøstheim (2001), hereafter referred to as KT. The theory of the present paper is different from the theory of $\mathrm{KT}$ in several aspects. In contrast to $\mathrm{KT}$, we consider a parametric nonstationary model. The absence of a kernel function makes it harder to prove existence of moments. On the other hand, the autoregressive structure makes it difficult to apply the local-time regression technique of Park and Phillips (2001) and Wang and Phillips (2009a, 2009b). The threshold structure and the splitting into two 
regimes are what makes it possible to employ some of the theory of KT in the present situation. The mathematical proofs of our theory are given in Appendix B.

\section{Estimation in parametric threshold autoregres- sive models}

We propose an ordinary least squares (OLS) estimation method for the unknown parameters $\alpha_{1}$ and $\alpha_{2}$ in Section 2.1. Some remarks about estimation of the $\tau$ parameter are given in Section 2.2.

\subsection{OLS estimation method and asymptotic theory}

Consider model (1.1). It is obvious that $\alpha_{1}$ and $\alpha_{2}$ can be estimated by the ordinary least squares estimators

$$
\begin{aligned}
& \widehat{\alpha}_{1}=\widehat{\alpha}_{1}(\tau)=\frac{\sum_{t=1}^{n} y_{t} y_{t-1} I\left[y_{t-1} \in C_{\tau}\right]}{\sum_{t=1}^{n} y_{t-1}^{2} I\left[y_{t-1} \in C_{\tau}\right]} \text { and } \\
& \widehat{\alpha}_{2}=\widehat{\alpha}_{2}(\tau)=\frac{\sum_{t=1}^{n} y_{t} y_{t-1} I\left[y_{t-1} \in D_{\tau}\right]}{\sum_{t=1}^{n} y_{t-1}^{2} I\left[y_{t-1} \in D_{\tau}\right]} .
\end{aligned}
$$

This implies that

$$
\begin{aligned}
\widehat{\alpha}_{1}-\alpha_{1} & =\frac{\sum_{t=1}^{n} e_{t} y_{t-1} I\left[y_{t-1} \in C_{\tau}\right]}{\sum_{t=1}^{n} y_{t-1}^{2} I\left[y_{t-1} \in C_{\tau}\right]} \text { and } \\
\widehat{\alpha}_{2}-1 & =\frac{\sum_{t=1}^{n} e_{t} y_{t-1} I\left[y_{t-1} \in D_{\tau}\right]}{\sum_{t=1}^{n} y_{t-1}^{2} I\left[y_{t-1} \in D_{\tau}\right]} .
\end{aligned}
$$

In order to establish an asymptotic distribution for each of the estimators, we first need to state some auxiliary results. Observe that model (1.1) can be written as

$$
y_{t}-y_{t-1}=\left(\alpha_{1}-1\right) y_{t-1} I\left[y_{t-1} \in C_{\tau}\right]+e_{t} \equiv u_{t}+e_{t}
$$

where $u_{t}=\left(\alpha_{1}-1\right) y_{t-1} I\left[y_{t-1} \in C_{\tau}\right]$.

Before further discussion, we need to introduce Lemma 2.1 below. As it is a special case of Lemma 3.1 below, we need only to prove Lemma 3.1 in Appendix B. 
Lemma 2.1 Let $\left\{y_{t}\right\}$ be generated by model (1.1). Then $\left\{y_{t}\right\}$ is a $\beta$-null recurrent Markov chain with $\beta=\frac{1}{2}$.

A $\beta$-null recurrent Markov chain possesses an invariant measure $\pi_{s}$ and there is a variable $T(n)$ keeping track of the number of regenerations at time $n$. Note that the definitions of $\pi_{s}(\cdot)$ and $T(n)$ are given in detail in Appendix A below. In this appendix, we have given a motivation for null recurrence in an econometric context and a very brief review of some key facts of the theory. If $C_{\tau}$ is compact, $T(n)$ may be taken to be proportional to the number of visits to $C_{\tau}$, as is seen from the remark at the end of this subsection. Let $\mu_{i}=\int_{-\infty}^{\infty} y^{i} I\left[y \in C_{\tau}\right] \pi_{s}(d y)$ for $i=1,2$. Then Lemma A.1(i) implies that the following limits hold almost surely,

$$
m_{u} \equiv \lim _{n \rightarrow \infty} \frac{1}{T(n)} \sum_{t=1}^{n} u_{t}=\lim _{n \rightarrow \infty} \frac{\left(\alpha_{1}-1\right)}{T(n)} \sum_{t=1}^{n} y_{t-1} I\left[y_{t-1} \in C_{\tau}\right]=\left(\alpha_{1}-1\right) \mu_{1} .
$$

It follows from Lemma A.2 in Appendix A and then Lemma 2.1 that as $n \rightarrow \infty$

$$
\frac{\sqrt{T([n r])}}{\sigma_{u}}\left(\frac{1}{T([n r])} \sum_{t=1}^{[n r]} u_{t}-m_{u}\right) \rightarrow_{D} B\left[M_{\beta}(r)\right]
$$

uniformly in $0 \leq r \leq 1$, where the symbol " $\rightarrow_{D}$ " means weak convergence in cadlag space (see, for example, the appendix of KT 2001), $\sigma_{u}^{2}=\mu_{2}-\mu_{1}^{2}$, and $M_{\beta}(t)$ is the Mittag-Leffler process as defined in $\mathrm{KT}$ (2001, p 388). Finally, $[x] \leq x$ is the largest integer part of $x$.

Let $\eta_{t}=u_{t}+e_{t}$. Using (2.6) and (2.7), it then follows from the continuous mapping theorem (Corollary 2 of Billingsley 1968, p. 31) and Lemma A.2 that as $n \rightarrow \infty$

$$
\begin{aligned}
Q_{n}(r) & \equiv \frac{1}{\sqrt{n}} \sum_{t=1}^{[n r]} \eta_{t}=\frac{1}{\sqrt{n}} \sum_{t=1}^{[n r]} u_{t}+\frac{1}{\sqrt{n}} \sum_{t=1}^{[n r]} e_{t} \\
& =\frac{\sigma_{u} \sqrt{T([n r])}}{\sqrt{T([n r])}} \frac{1}{\sigma_{u}}\left(\frac{1}{T([n r])} \sum_{t=1}^{[n r]} u_{t}-m_{u}\right)+\frac{T([n r])}{\sqrt{n}} m_{u} \\
& +\frac{1}{\sqrt{n}} \sum_{t=1}^{[n r]} e_{t}=\frac{\sigma_{u} \sqrt{T([n r])}}{\sqrt{n}} B\left[M_{\beta}(r)\right]+\frac{1}{\sqrt{n}} \sum_{t=1}^{[n r]} e_{t}+\frac{T([n r])}{\sqrt{n}} m_{u} \\
& +\quad o_{P}(1)=\frac{\sigma}{\sqrt{n} \sigma} \sum_{t=1}^{[n r]} e_{t}+\frac{T([n r])}{\sqrt{n}} m_{u}+o_{P}(1)
\end{aligned}
$$




$$
\rightarrow_{D} \quad \sigma B(r)+M_{\frac{1}{2}}(r) m_{u} \equiv Q(r)
$$

uniformly in $0<r \leq 1$, where Lemma A.4 in Appendix A has also been used.

This conclusion is summarized in Lemma 2.2.

Lemma 2.2 Let $\left\{y_{t}\right\}$ be generated by model (1.1). Then as $n \rightarrow \infty$

$$
Q_{n}(r)=\frac{1}{\sqrt{n}} \sum_{t=1}^{[n r]} u_{t}+\frac{1}{\sqrt{n}} \sum_{t=1}^{[n r]} e_{t} \rightarrow_{D} \sigma B(r)+M_{\frac{1}{2}}(r) m_{u} \equiv Q(r)
$$

Note that when $\mu_{1}=0$ and thus $m_{u}=0$, the contribution of $\left\{u_{t}\right\}$ to $\left\{y_{t}\right\}$ is asymptotically negligible. In this case, $\left\{y_{t}\right\}$ behaves like a random walk process.

We state the following lemma; its proof is given in Appendix B.

Lemma 2.3 Let model (1.1) hold. Then as $n \rightarrow \infty$

$$
\begin{aligned}
\frac{1}{T(n)} \sum_{t=1}^{n} y_{t-1}^{2} I\left[y_{t-1} \in C_{\tau}\right] & \rightarrow{ }_{P} \int_{-\infty}^{\infty} y^{2} I\left[y \in C_{\tau}\right] \pi_{s}(d y), \\
\frac{1}{n^{2}} \sum_{t=1}^{n} y_{t-1}^{2} I\left[y_{t-1} \in D_{\tau}\right] & \stackrel{d}{\longrightarrow} \int_{0}^{1} Q^{2}(r) d r \\
\frac{1}{\sqrt{T(n)}} \sum_{t=1}^{n} y_{t-1} e_{t} I\left[y_{t-1} \in C_{\tau}\right] & \stackrel{d}{\longrightarrow} N\left(0, \sigma_{1}^{2}\right), \\
\frac{1}{n} \sum_{t=1}^{n} y_{t-1} e_{t} I\left[y_{t-1} \in D_{\tau}\right] & \stackrel{d}{\longrightarrow} \frac{1}{2}\left(Q^{2}(1)-\sigma^{2}\right),
\end{aligned}
$$

where the symbol " $\stackrel{d}{\longrightarrow}$ " denotes convergence in distribution, $\sigma_{1}^{2}=\sigma^{2} \int_{-\infty}^{\infty} y^{2} I[y \in$ $\left.C_{\tau}\right] \pi_{s}(d y)$ and $Q(r)=\sigma B(r)+\left(\alpha_{1}-1\right) \mu_{1} M_{\frac{1}{2}}(r)$.

We now state the main results of this section; its proof is given in Appendix B.

Theorem 2.1 Let model (1.1) hold. Then as $n \rightarrow \infty$

$$
\begin{aligned}
\sqrt{T(n)}\left(\widehat{\alpha}_{1}-\alpha_{1}\right) & \stackrel{d}{\longrightarrow} N\left(0, \sigma^{4} \sigma_{1}^{-2}\right), \\
n\left(\widehat{\alpha}_{2}-1\right) & \stackrel{d}{\longrightarrow} \frac{\left(Q^{2}(1)-\sigma^{2}\right)}{2 \int_{0}^{1} Q^{2}(r) d r} .
\end{aligned}
$$

Note that $Q(r)=\sigma B(r)$ when $\mu_{1}=0$. This implies that the asymptotic theory for $\widehat{\alpha}_{2}$ is the same as that for the unit-root case when $\mu_{1}=\int_{-\infty}^{\infty} y I\left[y \in C_{\tau}\right] \pi_{s}(d y)=0$, 
i.e., $\left\{y_{t}\right\}$ has some symmetrical structure in the stationary regime. In this symmetrical case, the asymptotic distribution in (2.15) corresponds to the main result in Theorem 2.1 of Liu, Ling and Shao (2009). In the more general case, it is harder to interpret the right hand side of (2.15). The Mittag-Leffler variable $M_{\beta}(r)$ represents the distributional limit of a scaled version of $T([n r])$. As shown in Lemma 3.2 of $\mathrm{KT}$ and then Theorem 2.1 of Wang and Phillips (2009a), we have $\frac{T(n)}{\sqrt{n}} \rightarrow_{P} L_{B}(1,0)$ as $n \rightarrow \infty$, where $L_{B}(t, 0)=\lim _{\epsilon \rightarrow 0} \frac{1}{2 \epsilon} \int_{0}^{t} I[|B(s)|<\epsilon] d s$ is the local-time process of the Brownian motion process $B(r)$. As a consequence, one may see that $T(n)$ is asymptotically equivalent to $\sqrt{n} L_{B}(1,0)$, which may be more computationally usable. In practice, it will probably be better to simulate the right hand side of (2.15) by bootstrapping the residuals of the model. In fact, a bootstrap procedure was used in Gao et al (2009a, 2009b) to conduct unit root type test in a nonlinear and nonstationary environment.

REMARK 2.1. Theorem 2.1 shows that the rate of convergence of $\widehat{\alpha}_{1}$ to $\alpha_{1}$ is proportional to $\sqrt{T(n)}$ while the rate of convergence of $\widehat{\alpha}_{2}$ to 1 is proportional to $n$. According to Lemmas 2.1 and 3.4 and Theorem 3.2 of $\mathrm{KT}, T(n)$ behaves asymptotically as the Mittag-Leffler variable $M_{\frac{1}{2}}(\cdot)$ and in the limit can be associated with the deterministic convergence rate of $n^{-\frac{1}{2}}$. As mentioned above, our results can be translated to local-time terminology and are threshold autoregressive counterparts of the results in Phillips (1987), and Park and Phillips (2001). The results of those papers were for the nonlinear and nonstationary regression case and it is not clear whether the local time techniques used there can be extended to an autoregressive situation. Note that $T(n)$ may be replaced by $\frac{T_{C}(n)}{\pi_{s}\left(1_{C}\right)}$ (Lemma 3.6 of Karlsen and Tjøstheim 2001), where $T_{C}(n)$ is the number of visits to a small set $C$, which may be taken to be a subset of $C_{\tau}$ or $C_{\tau}$ itself if it is compact.

\subsection{Remarks about estimation of the $\tau$ parameter}

In both theory and practice, estimation of the $\tau$ parameter is of interest and importance.

Let $\widehat{e}_{t}(\tau)=y_{t}-\widehat{\alpha}_{1} y_{t-1} I\left[y_{t-1} \in C_{\tau}\right]-\widehat{\alpha}_{2} y_{t-1} I\left[y_{t-1} \in D_{\tau}\right]$ and then define the estimated variance by

$$
\widehat{\sigma}^{2}(\tau)=\frac{1}{n} \sum_{t=1}^{n} \widehat{e}_{t}^{2}(\tau) .
$$


The $\tau$ parameter can then be estimated by

$$
\widehat{\tau}=\arg \min _{\text {over all } \tau} \widehat{\sigma}^{2}(\tau)
$$

In both the stationary and nonstationary unit-root cases, asymptotic properties of $\widehat{\tau}$ have been discussed (see, for example, Pham, Chan and Tong 1991; Chan 1993; Hansen 2000). In the paper by Chan (1993), the author shows that the rate of convergence of $\widehat{\tau}$ to $\tau$ can be as fast as the super-rate of $n$, see also Li and Ling (2011). Our simulation study in Section 4, however, suggests that the rate of convergence of $\widehat{\tau}$ to $\tau$ may be related to $T(n)$, as will also be pointed out in the discussion below.

Studying asymptotic properties for $\widehat{\tau}$ in detail for the model we are considering requires a separate investigation, since even in the stationary case the theory is quite complex (see, for example, Chan 1993). In the present paper, we will only indicate how Chan's proof of consistency can be extended and comment on the rate that can be expected.

Chan (1993) restricts himself to the case of a single threshold $\tau$, so that there is a stationary regime to the left of $\tau$ and another stationary regime to the right of $\tau$. In our discussion we will use the same simplification but with one of the regimes being a unit root regime. Moreover, since we only look at the first order case, we take the threshold variable $y_{t-d}$ to be $y_{t-1}$.

Chan (1993) makes use of ergodicity in his proof, which we do not have in our case, but his proof of consistency can nevertheless be adapted to our situation by noting that

$$
L(\theta)=\sum_{t=1}^{n}\left(y_{t}-E_{\theta}\left(y_{t} \mid \mathcal{F}_{t-1}\right)\right)^{2}
$$

can be decomposed using the existence of the regeneration mechanism for a null recurrent process, such that (see (A.3) and (A.4) of Appendix A)

$$
L(\theta)=U_{0}+\sum_{k=1}^{T(n)} U_{k}+U_{(n)}
$$

Here, $\theta$ is the parameter composed of the AR coefficients and the threshold, with $\theta$ belonging to a parameter space $\Theta$. Moreover, $E_{\theta}\left(\cdot \mid \mathcal{F}_{t-1}\right)$ is the conditional expectation 
with respect to the $\sigma$-field $\mathcal{F}_{t-1}$ generated by $\left\{y_{s}, 1 \leq s \leq t-1\right\}$, and

$$
U_{k}=U_{k}(g, \theta)=\sum_{t=\tau_{k-1}+1}^{\tau_{k}} g\left(y_{t}, y_{t+1}, \theta\right)
$$

with $g\left(y_{t-1}, y_{t}, \theta\right)=\left(y_{t}-E_{\theta}\left(y_{t} \mid \mathcal{F}_{t-1}\right)\right)^{2}$, and where the $\tau_{k}$ 's are regeneration times. Finally, $U_{0}$ and $U_{(n)}$ in (2.18) are a starting term and a residual that can be neglected as $n \rightarrow \infty$. The sequence $\left\{U_{k}\right\}$ consists of random variables that are identically distributed and are 1-dependent. It essentially takes the place of the ergodic process $\left\{y_{t}\right\}$ in Chan's proof and of course $\left\{U_{k}\right\}$ trivially fulfills the stationarity and ergodicity requirement of his Theorem 1, where strong consistency is proved.

The decomposition of (3.2) of Chan (1993) can now be written as

$$
\sum_{t=\tau_{k-1}+1}^{\tau_{k}}\left(y_{t}-E_{\theta}\left(y_{t} \mid \mathcal{F}_{t-1}\right)\right)^{2}=R_{1 k}(\theta)+R_{2 k}(\theta)+R_{3 k}(\theta)+R_{4 k}(\theta)
$$

with

$$
\begin{aligned}
& R_{1 k}(\theta)=\sum_{t=\tau_{k-1}+1}^{\tau_{k}}\left(y_{t}-\beta_{1} y_{t-1}\right)^{2} I\left(y_{t-1} \leq z, y_{t-1} \leq \tau\right), \\
& R_{2 k}(\theta)=\sum_{t=\tau_{k-1}+1}^{\tau_{k}}\left(y_{t}-\beta_{1} y_{t-1}\right)^{2} I\left(y_{t-1} \leq z, y_{t-1}>\tau\right), \\
& R_{3 k}(\theta)=\sum_{t=\tau_{k-1}+1}^{\tau_{k}}\left(y_{t}-\beta_{2} y_{t-1}\right)^{2} I\left(y_{t-1}>z, y_{t-1} \leq \tau\right), \\
& R_{4 k}(\theta)=\sum_{t=\tau_{k-1}+1}^{\tau_{k}}\left(y_{t}-\beta_{2} y_{t-1}\right)^{2} I\left(y_{t-1}>z, y_{t-1}>\tau\right),
\end{aligned}
$$

where $\beta_{1}, \beta_{2}$ and $z$ are neighboring points of the true values $\alpha_{1}, \alpha_{2}$ and $\tau$ in the parameter space $\Theta$. Next, $R_{i k}(\theta), i=1, \cdots, 4$ can themselves be decomposed analogously to (3.3) in Chan (1993) by adding and substracting the true AR parameters $\alpha_{1}$ and $\alpha_{2}$. With these changes, the proof of Lemma 1 in Chan (1993) can be carried through. Moreover, in the proof of his claim 1, $\sigma_{\theta}^{2}$ can be replaced by $E\left[\sum_{t=\tau_{k-1}+1}^{\tau_{k}}\left(y_{t}-E_{\theta_{0}}\left(y_{t} \mid \mathcal{F}_{t-1}\right)\right)^{2}\right]$ with $\theta_{0}=\left(\alpha_{1}, \alpha_{2}\right)$, and one has to introduce a truncation variable to ensure the existence of $E\left[R_{i k}(\theta)\right]$. The rest of the proof of consistency can be carried out along the lines of Chan (1993). 
Chan (1993) obtains a rate for $\widehat{\tau}-\tau$ of order $n^{-1}$. By the decomposition in (2.18) we effectively introduce a 1-dependent process where $T(n)$ can be taken as the number of observations. We could therefore possibly expect a rate for $\widehat{\tau}-\tau$ of order $T^{-1}(n)$ which can be associated with $n^{-\frac{1}{2}}$. This is in agreement with the finite sample results for Case A of Example 4.3 below, which is an example where a threshold of this type was investigated by simulation. The finite sample rate for the other examples, where two thresholds are involved, is slower. It should be kept in mind, though, that the association of $T(n)$ with $\sqrt{n}$ is itself an asymptotic result (see, for example, Lemma 3.4 and Theorem 3.2 of KT. For a finite $n, T(n)$ will certainly depend on the set $C_{\tau}$. Rigorous conditions and results about the rate and indeed about the asymptotic distribution would require an extension of Propositions 1 and 2 as well as Theorem 2 of Chan (1993). This is far from trivial and would require a separate paper.

\section{Estimation in semiparametric threshold autore- gressive models}

This section considers a semiparametric threshold autoregressive (SEMI-TAR) model of the form

$$
\begin{aligned}
y_{t} & =g\left(y_{t-1}\right) I\left[y_{t-1} \in C_{\tau}\right]+\alpha y_{t-1} I\left[y_{t-1} \in D_{\tau}\right]+e_{t} \\
& =\left\{\begin{array}{cl}
g\left(y_{t-1}\right)+e_{t} & \text { if } y_{t-1} \in C_{\tau}, \\
\alpha y_{t-1}+e_{t} & \text { if } y_{t-1} \in D_{\tau},
\end{array}\right.
\end{aligned}
$$

where $C_{\tau}$ and $D_{\tau}$ are as defined in (1.1), $g(x)$ is an unknown and bounded function when $x \in C_{\tau}, \alpha=1$, and $\left\{e_{t}\right\}$ is the same as assumed in (1.1). Let $y_{0}=0$. Model (3.1) may be used to detect and then estimate structural change from a nonlinear 'stationary' regime to a linear 'nonstationary' regime.

While the special case of $\alpha=1$ of model (3.1) has been mentioned in Example 3.1 of $\mathrm{KT}$ as an example of a null recurrent process, asymptotic estimation theory for model (3.1) has not been studied in the literature. Existing results for the stationary nonlinear time series models (Tong 1990; Fan and Yao 2003; Gao 2007; Teräsvirta, 
Tjøstheim and Granger 2010) are also not directly applicable to study such SEMITAR models. Our interest is to study asymptotic behavior of both a nonparametric estimator of $g(\cdot)$ and an OLS estimator of $\alpha$.

In order to establish consistent estimates for $g(\cdot)$ and $\alpha$, we need to introduce the following assumption.

Assumption 3.1 (i) The invariant measure $\pi_{s}$ of $\left\{y_{t}\right\}$ has a locally continuous density $p_{s}(y)$ that is locally strictly positive; that is, $p_{s}(y)>0$ for all $y \in R^{1}$.

(ii) Let $g(y)$ be twice differentiable and the second derivative $g^{\prime \prime}(y)$ be continuous at all $y \in R^{1}$.

(iii) Let $K(\cdot)$ be a symmetric probability kernel function with compact support $C(K)$. The bandwidth parameter $h$ satisfies $\lim _{n \rightarrow \infty} h=0, \lim _{n \rightarrow \infty} n h=\infty$ and $\lim \sup _{n \rightarrow \infty} n^{1+\delta_{0}} h^{6}<\infty$ for some $0<\delta_{0}<\frac{1}{2}$.

(iv) In case $C_{\tau}$ is not compact, i.e. $C_{\tau}=(-\infty, \tau]$ or $C_{\tau}=[\tau, \infty),|g(y)| \leq c_{g}|y|$ with $0<c_{g}<1$ as $|y| \rightarrow \infty$.

Conditions in Assumption 3.1(i)(ii)(iii) are quite mild conditions (see, for example, Assumptions $B_{0}-B_{3}$ of KT. Condition 3.1(iv) is to secure stationary type behavior on $C_{\tau}$.

We need the following lemma; its proof is given in Appendix A below.

Lemma 3.1 Let $\left\{y_{t}\right\}$ be generated by model (3.1). If Assumption 3.1(i)(ii)(iv) holds, then $\left\{y_{t}\right\}$ is a $\beta$-null recurrent Markov chain with $\beta=\frac{1}{2}$.

Similarly to (2.5), we have

$$
y_{t}-y_{t-1}=\left(g\left(y_{t-1}\right)-y_{t-1}\right) I\left[y_{t-1} \in C_{\tau}\right]+e_{t} \equiv v_{t}+e_{t} .
$$

Let $\mu_{g}=\int_{-\infty}^{\infty} g(y) I\left[y \in C_{\tau}\right] \pi_{s}(d y)$. Then Lemma A.1(i) below implies that the following limits hold almost surely,

$$
g_{v} \equiv \lim _{n \rightarrow \infty} \frac{1}{T(n)} \sum_{t=1}^{n} v_{t}=\lim _{n \rightarrow \infty} \frac{1}{T(n)} \sum_{t=1}^{n}\left(g\left(y_{t-1}\right)-y_{t-1}\right) I\left[y_{t-1} \in C_{\tau}\right]=\mu_{g}-\mu_{1}
$$

where $\mu_{1}$ is as defined in (2.6).

We state the following lemma; its proof is similar to equations (2.7)-(2.9). 
Lemma 3.2 Let $\left\{y_{t}\right\}$ be generated by model (3.1). If Assumption 3.1(i)(ii)(iv) holds, then as $n \rightarrow \infty$

$$
P_{n}(r) \equiv \frac{1}{\sqrt{n}} \sum_{t=1}^{[n r]} e_{t}+\frac{1}{\sqrt{n}} \sum_{t=1}^{[n r]} v_{t} \rightarrow_{D} \sigma B(r)+M_{\frac{1}{2}}(r) g_{v} \equiv P(r) .
$$

Let $K(\cdot)$ be a probability kernel function and $h$ be a bandwidth parameter satisfying Assumption 3.1(iii) above. It is obvious that $g(y)$ and $\alpha$ can be estimated by

$$
\begin{aligned}
\widehat{g}(y) & =\widehat{g}(y, \tau)=\frac{\sum_{t=1}^{n} K\left(\frac{y-y_{t-1}}{h}\right) y_{t} I\left[y_{t-1} \in C_{\tau}\right]}{\sum_{t=1}^{n} K\left(\frac{y-y_{t-1}}{h}\right) I\left[y_{t-1} \in C_{\tau}\right]} \text { and } \\
\widehat{\alpha} & =\widehat{\alpha}(\tau)=\frac{\sum_{t=1}^{n} y_{t} y_{t-1} I\left[y_{t-1} \in D_{\tau}\right]}{\sum_{t=1}^{n} y_{t-1}^{2} I\left[y_{t-1} \in D_{\tau}\right]},
\end{aligned}
$$

which imply that

$$
\begin{aligned}
\widehat{g}(y)-g(y) & =\frac{\sum_{t=1}^{n} K\left(\frac{y-y_{t-1}}{h}\right)\left(g\left(y_{t-1}\right)-g(y)\right) I\left[y_{t-1} \in C_{\tau}\right]}{\sum_{t=1}^{n} K\left(\frac{y-y_{t-1}}{h}\right) I\left[y_{t-1} \in C_{\tau}\right]} \\
& +\frac{\sum_{t=1}^{n} K\left(\frac{y-y_{t-1}}{h}\right) e_{t} I\left[y_{t-1} \in C_{\tau}\right]}{\sum_{t=1}^{n} K\left(\frac{y-y_{t-1}}{h}\right) I\left[y_{t-1} \in C_{\tau}\right]}, \\
\widehat{\alpha}-1 & =\frac{\sum_{t=1}^{n} e_{t} y_{t-1} I\left[y_{t-1} \in D_{\tau}\right]}{\sum_{t=1}^{n} y_{t-1}^{2} I\left[y_{t-1} \in D_{\tau}\right]} .
\end{aligned}
$$

We now state the main results of this section; its proof is given in Appendix B.

TheOREM 3.1 Let both model (3.1) and Assumption 3.1 hold. Then as $n \rightarrow \infty$

$$
\begin{aligned}
\sqrt{\sum_{t=1}^{n} K\left(\frac{y-y_{t-1}}{h}\right) I\left[y \in C_{\tau}\right]}(\widehat{g}(y)-g(y)) & \stackrel{d}{\longrightarrow} N\left(0, \sigma_{2}^{2}\right), \\
n(\widehat{\alpha}-1) & \stackrel{d}{\longrightarrow} \frac{\left(P^{2}(1)-\sigma^{2}\right)}{2 \int_{0}^{1} P^{2}(r) d r},
\end{aligned}
$$

where $\sigma_{2}^{2}=\sigma^{2} \int K^{2}(u) d u$ and $P(r)=\sigma B(r)+M_{\frac{1}{2}}(r) g_{v}$. Note that $P(r)=\sigma B(r)$ when $g_{v}=0$.

Remark 3.1. Compared with Theorem 2.1, Theorem 3.1 shows that while the parameter estimator $\widehat{\alpha}$ has the same asymptotic distribution as that of $\widehat{\alpha}_{2}$, the nonparametric estimator $\widehat{g}(\cdot)$ as expected has a rate of convergence slower than its para- 
metric counterpart $\widehat{\alpha}_{1}$. In addition, Theorem 3.1 shows that the rate of convergence of $\widehat{g}(\cdot)$ is also slower than that of the corresponding nonparametric kernel estimator for the stationary case, as shown in KT, Karlsen, MyKlebust and Tjøstheim (2007), and Wang and Phillips (2009a; 2009b). For the interpretations and possible computation of the right hand side of (3.6), the same comments as for (2.15) apply.

Other closely related papers in the field of nonparametric and semiparametric regression estimation and specification testing involving nonstationary time series include Chen, Gao and Li (2008), Cai, Li and Park (2009), and Gao et al (2009a, 2009b).

\section{Examples of implementation}

This section gives several examples to evaluate the finite-sample performance of the proposed estimation method in several different cases. There are four simulation examples and one real date example.

Consider a general threshold autoregressive (TAR) model of the form

$$
y_{t}=\alpha_{1} y_{t-1} I\left[y_{t-1} \in C_{\tau}\right]+\alpha_{2} y_{t-1} I\left[y_{t-1} \in C_{\tau}^{c}\right]+e_{t}, 1 \leq t \leq n
$$

where $\tau=\left(\tau_{1}, \tau_{2}\right), C_{\tau}=\left[\tau_{1}, \tau_{2}\right]$ for $-\infty<\tau_{1}<\tau_{2}<\infty$ with both $\tau_{1}$ and $\tau_{2}$ being the threshold parameters, $C_{\tau}^{c}=\left(-\infty, \tau_{1}\right) \cup\left(\tau_{2}, \infty\right)$, and $\left\{e_{t}\right\}$ is assumed to be a sequence of independent and normally distributed random errors with $E\left[e_{1}\right]=0$ and $\sigma^{2}=E\left[e_{1}^{2}\right]=$ 1. That is, $e_{t} \sim N(0,1)$. Let $y_{0}=0$.

The unknown parameters $\alpha_{1}, \alpha_{2}$ and $\tau$ are estimated by the ordinary least squares estimators:

$$
\begin{aligned}
\widetilde{\alpha}_{1} & =\widehat{\alpha}_{1}(\widehat{\tau})=\frac{\sum_{t=1}^{n} y_{t} y_{t-1} I\left[y_{t-1} \in C_{\widehat{\tau}}\right]}{\sum_{t=1}^{n} y_{t-1}^{2} I\left[y_{t-1} \in C_{\widehat{\tau}}\right]}, \\
\widetilde{\alpha}_{2} & =\widehat{\alpha}_{2}(\widehat{\tau})=\frac{\sum_{t=1}^{n} y_{t} y_{t-1} I\left[y_{t-1} \in C_{\widehat{\tau}}^{c}\right]}{\sum_{t=1}^{n} y_{t-1}^{2} I\left[y_{t-1} \in C_{\widehat{\tau}}^{c}\right]}, \\
\widehat{\tau} & =\arg \min _{\text {over all } \tau} \widehat{\sigma}^{2}(\tau)
\end{aligned}
$$

where $\widehat{\sigma}^{2}(\tau)=\frac{1}{n} \sum_{t=1}^{n}\left(y_{t}-\widehat{\alpha}_{1}(\tau) y_{t-1} I\left[y_{t-1} \in C_{\tau}\right]-\widehat{\alpha}_{2}(\tau) y_{t-1} I\left[y_{t-1} \in C_{\tau}^{c}\right]\right)^{2}$. Let $\widehat{\tau}=$ $\left(\widehat{\tau}_{1}, \widehat{\tau}_{2}\right)$ for the asymmetrical case with $\tau_{2} \neq-\tau_{1}$. 
Example 4.1 consider a symmetrical case of the form $C_{\tau}=[-\tau, \tau]$. An asymmetrical bounded case where $C_{\tau}=\left[\tau_{1}, \tau_{2}\right]$ is discussed in Example 4.2 below. Example 4.3 examines the unbounded case where $C_{\tau}=(-\infty, \tau]$. Throughout Examples 4.1-4.3 below, we consider both the cases of $\mathrm{A}:\left|\alpha_{1}\right|<1$ and $\mathrm{B}:\left|\alpha_{1}\right|>1$.

- Consider the case of $n=1000,2000,5000$ and 10000. Let $N=1000$ be the number of replications and $\widetilde{\alpha}_{i}(j)$ and $\widehat{\tau}(j)$ be the respective value of $\widetilde{\alpha}_{i}$ and $\widehat{\tau}$ at the $j$-th replication.

- Calculate the standard deviations of the form

$$
\operatorname{std}\left(\widetilde{\alpha}_{i}\right)=\sqrt{\frac{1}{N-1} \sum_{j=1}^{N}\left(\widetilde{\alpha}_{i}(j)-\overline{\widetilde{\alpha}}_{i}\right)^{2}} \text { and } \operatorname{std}(\widehat{\tau})=\sqrt{\frac{1}{N-1} \sum_{j=1}^{N}(\widehat{\tau}(j)-\overline{\widehat{\tau}})^{2}}
$$

for $i=1,2$ and Cases A and B separately under $N=1000$, where $\overline{\widetilde{\alpha}}_{i}=\frac{1}{N} \sum_{j=1}^{N} \widetilde{\alpha}_{i}(j)$ and $\bar{\tau}=\frac{1}{N} \sum_{j=1}^{N} \widehat{\tau}(j)$.

- Calculate the biases of the form

$$
\operatorname{Bias}\left(\widetilde{\alpha}_{i}\right)=\frac{1}{N} \sum_{j=1}^{N}\left(\widetilde{\alpha}_{i}(j)-\alpha_{i}\right) \text { and } \operatorname{Bias}(\widehat{\tau})=\frac{1}{N} \sum_{j=1}^{N}(\widehat{\tau}(j)-\tau)
$$

for $i=1,2$ and Cases A and B separately under $N=1000$.

- Calculate the Root Mean Squared Errors of the form

$$
\operatorname{RMSE}\left(\widetilde{\alpha}_{i}\right)=\sqrt{\frac{1}{N} \sum_{j=1}^{N}\left(\widetilde{\alpha}_{i}(j)-\alpha_{i}\right)^{2}} \text { and } \operatorname{RMSE}(\widehat{\tau})=\sqrt{\frac{1}{N} \sum_{j=1}^{N}(\widehat{\tau}(j)-\tau)^{2}}
$$

for $i=1,2$ and Cases A and B separately under $N=1000$.

Example 4.1 Consider a symmetrical (bounded) threshold autoregressive (TAR) model of the form

$$
y_{t}=\alpha_{1} y_{t-1} I\left[\left|y_{t-1}\right| \leq \tau\right]+\alpha_{2} y_{t-1} I\left[\left|y_{t-1}\right|>\tau\right]+e_{t}, 1 \leq t \leq n
$$

This example then considers the following cases.

- Case A: $\alpha_{1}=\frac{1}{2}, \alpha_{2}=1$ and $\tau=2.5$; and 
- Case B: $\alpha_{1}=\frac{3}{2}, \alpha_{2}=1$ and $\tau=2$.

The simulated results for Example 4.1 are given in Table 4.1 below.

Table 4.1 Simulation Results for Case A and Case B

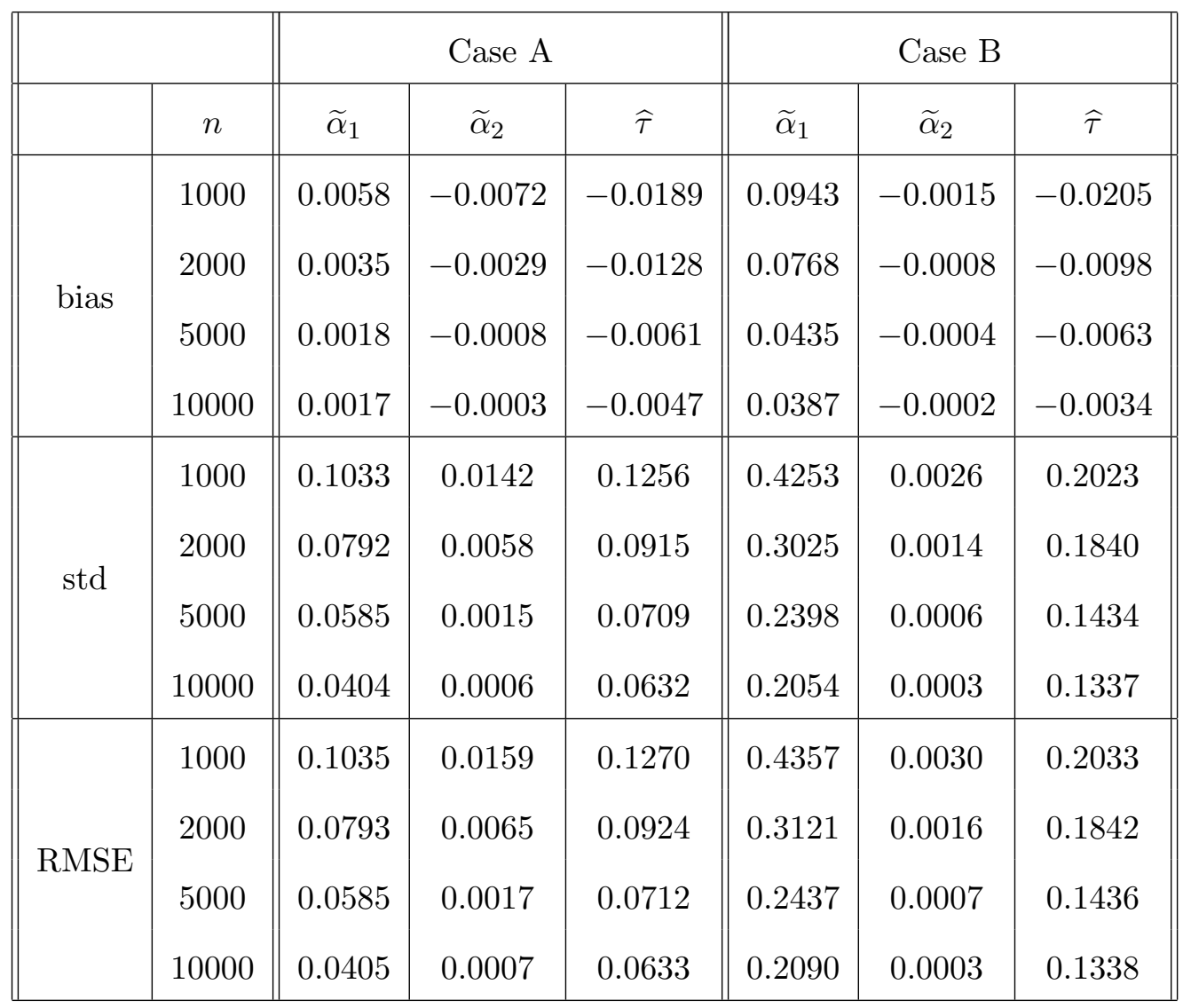

Table 4.1 supports the rate results of Theorem 2.1 if standard errors for the various observation sizes are compared. Also, for this model as well as for the following models, the number of samples in the stationary regime should be of order $\sqrt{n}$. This was found to be the case for the experiments conducted by us. Case B has larger standard errors for $\widehat{\alpha}_{1}$ and smaller standard errors for $\widehat{\alpha}_{2}$, because the explosive behavior on $C_{\tau}$ in this case leads to more frequent stays in the random walk regime.

Example 4.2 Consider an asymmetrical (bounded) threshold autoregressive (TAR) model of the form

$$
y_{t}=\alpha_{1} y_{t-1} I\left[y_{t-1} \in C_{\tau}\right]+\alpha_{2} y_{t-1} I\left[y_{t-1} \in C_{\tau}^{c}\right]+e_{t}, 1 \leq t \leq n
$$

We are then interested in the following cases: 
- Case A: $\alpha_{1}=\frac{1}{2}, \alpha_{2}=1, \tau_{1}=-3$ and $\tau_{2}=2.5$; and

- Case B: $\alpha_{1}=\frac{3}{2}, \alpha_{2}=1, \tau_{1}=-1.5$ and $\tau_{2}=1$.

The simulated results for Example 4.2 are given in Table 4.2 below. Similarly to Table 4.1, Table 4.2 also demonstrates that the proposed estimation method still works well numerically even when two truncation parameters are involved in the model.

Example 4.3 Consider a threshold autoregressive (TAR) model with unbounded $C_{\tau}$ of the form

$$
y_{t}=\alpha_{1} y_{t-1} I\left[y_{t-1} \leq \tau\right]+\alpha_{2} y_{t-1} I\left[y_{t-1}>\tau\right]+e_{t}, 1 \leq t \leq n
$$

This example also considers the following cases:

- Case A: $\alpha_{1}=\frac{1}{2}, \alpha_{2}=1, \tau=3$; and

- Case B: $\alpha_{1}=\frac{3}{2}, \alpha_{2}=1, \tau=3$.

The simulated results for Example 4.3 are given in Table 4.3 below.

Table 4.3 again supports the rate results of Theorem 2.1. Note that Case B is not covered by Assumption 3.1(iv), but it works well because the process "explodes" from $(-\infty, \tau]$ into the random walk part $[\tau, \infty)$. 
Table 4.2 Simulation Results for Cases A and B

\begin{tabular}{|c|c|c|c|c|c|}
\hline \multicolumn{6}{|c|}{ Case A } \\
\hline & $n$ & $\widetilde{\alpha}_{1}$ & $\widetilde{\alpha}_{2}$ & $\widehat{\tau}_{1}$ & $\widehat{\tau}_{2}$ \\
\hline \multirow{4}{*}{ bias } & 1000 & 0.0019 & -0.0110 & -0.1158 & -0.0214 \\
\hline & 2000 & 0.0070 & -0.0045 & -0.0753 & -0.0202 \\
\hline & 5000 & -0.0001 & -0.0012 & -0.0389 & -0.0089 \\
\hline & 10000 & -0.0011 & -0.0004 & -0.0333 & -0.0075 \\
\hline \multirow{4}{*}{ std } & 1000 & 0.1308 & 0.0200 & 0.2536 & 0.1481 \\
\hline & 2000 & 0.0890 & 0.0086 & 0.2089 & 0.1208 \\
\hline & 5000 & 0.0704 & 0.0023 & 0.1581 & 0.0950 \\
\hline & 10000 & 0.0367 & 0.0009 & 0.1345 & 0.0663 \\
\hline \multirow{4}{*}{ RMSE } & 1000 & 0.1308 & 0.0228 & 0.2788 & 0.1496 \\
\hline & 2000 & 0.0893 & 0.0097 & 0.2220 & 0.1225 \\
\hline & 5000 & 0.0704 & 0.0026 & 0.1628 & 0.0954 \\
\hline & 10000 & 0.0367 & 0.0010 & 0.1386 & 0.0667 \\
\hline \multicolumn{6}{|c|}{ Case B } \\
\hline \multirow{4}{*}{ bias } & 1000 & 0.2047 & -0.0017 & -0.0433 & -0.1126 \\
\hline & 2000 & 0.0924 & -0.0009 & -0.0329 & -0.0764 \\
\hline & 5000 & 0.0990 & -0.0004 & -0.0129 & -0.0623 \\
\hline & 10000 & 0.0896 & -0.0002 & -0.0035 & -0.0556 \\
\hline \multirow{4}{*}{ std } & 1000 & 0.8657 & 0.0030 & 0.2695 & 0.3115 \\
\hline & 2000 & 0.7072 & 0.0015 & 0.2536 & 0.3013 \\
\hline & 5000 & 0.5993 & 0.0006 & 0.2180 & 0.2712 \\
\hline & 10000 & 0.3156 & 0.0003 & 0.1886 & 0.2535 \\
\hline \multirow{4}{*}{ RMSE } & 1000 & 0.8896 & 0.0034 & 0.2730 & 0.3313 \\
\hline & 2000 & 0.7132 & 0.0018 & 0.2558 & 0.3108 \\
\hline & 5000 & 0.6074 & 0.0007 & 0.2184 & 0.2783 \\
\hline & 10000 & 0.3280 & 0.0003 & 0.1886 & 0.2596 \\
\hline
\end{tabular}


Table 4.3 Simulation Results for Case A and Case B

\begin{tabular}{|c|c|c|c|c|c|c|c|}
\hline & & \multicolumn{3}{|c|}{ Case A } & \multicolumn{3}{|c|}{ Case B } \\
\hline & $n$ & $\widetilde{\alpha}_{1}$ & $\widetilde{\alpha}_{2}$ & $\widehat{\tau}$ & $\widetilde{\alpha}_{1}$ & $\widetilde{\alpha}_{2}$ & $\widehat{\tau}$ \\
\hline \multirow{4}{*}{ bias } & 1000 & -0.0013 & -0.0740 & -0.0601 & 0.0479 & -0.0014 & -0.0227 \\
\hline & 2000 & -0.0009 & -0.0307 & -0.0180 & 0.0357 & -0.0007 & -0.0243 \\
\hline & 5000 & 0.0009 & -0.0082 & -0.0067 & 0.0332 & -0.0002 & -0.0141 \\
\hline & 10000 & 0.0001 & -0.0030 & -0.0020 & 0.0290 & -0.0001 & -0.0158 \\
\hline \multirow{4}{*}{ std } & 1000 & 0.0392 & 0.1199 & 0.1633 & 0.1732 & 0.0031 & 0.1713 \\
\hline & 2000 & 0.0365 & 0.0536 & 0.1065 & 0.1550 & 0.0013 & 0.1594 \\
\hline & 5000 & 0.0262 & 0.0161 & 0.0715 & 0.1327 & 0.0004 & 0.1409 \\
\hline & 10000 & 0.0134 & 0.0061 & 0.0442 & 0.1105 & 0.0002 & 0.1181 \\
\hline \multirow{4}{*}{ RMSE } & 1000 & 0.0392 & 0.1409 & 0.1740 & 0.1797 & 0.0034 & 0.1728 \\
\hline & 2000 & 0.0365 & 0.0618 & 0.1080 & 0.1591 & 0.0014 & 0.1612 \\
\hline & 5000 & 0.0263 & 0.0181 & 0.0718 & 0.1368 & 0.0005 & 0.1416 \\
\hline & 10000 & 0.0134 & 0.0068 & 0.0443 & 0.1143 & 0.0002 & 0.1192 \\
\hline
\end{tabular}

In the following example, we consider a semiparametric threshold autoregressive model and then study the finite sample performance of the proposed semiparametric estimation method.

Example 4.4 Consider a semiparametric threshold autoregressive (SEMI-TAR) model of the form

$$
y_{t}=g\left(y_{t-1}\right) I\left[\left|y_{t-1}\right| \leq \tau\right]+\alpha y_{t-1} I\left[\left|y_{t-1}\right|>\tau\right]+e_{t},
$$

where $\tau=2.5$ and $e_{t} \sim N(0,1)$. Let $y_{0}=0$.

Let $K(x)=\frac{1}{2} I_{[-1,1]}(x)$. We then estimate $g(y)$ and $\alpha$ by

$$
\begin{aligned}
\widehat{g}(y, \widehat{\tau}) & =\frac{\sum_{t=1}^{n} K\left(\frac{y-y_{t-1}}{\widehat{h}_{\mathrm{cv}}}\right) y_{t} I\left[\left|y_{t-1}\right| \leq \widehat{\tau}\right]}{\sum_{t=1}^{n} K\left(\frac{y-y_{t-1}}{\widehat{h}_{\mathrm{cv}}}\right) I\left[\left|y_{t-1}\right| \leq \widehat{\tau}\right]}, \\
\widetilde{\alpha} & =\widehat{\alpha}(\widehat{\tau})=\frac{\sum_{t=1}^{n} y_{t} y_{t-1} I\left[\left|y_{t-1}\right|>\widehat{\tau}\right]}{\sum_{t=1}^{n} y_{t-1}^{2} I\left[\left|y_{t-1}\right|>\widehat{\tau}\right]} \\
\widehat{\tau} & =\arg \min _{\text {over all } \tau} \widehat{\sigma}^{2}(\tau),
\end{aligned}
$$


where $\widehat{\sigma}^{2}(\tau)=\frac{1}{n} \sum_{t=1}^{n}\left(y_{t}-\widehat{g}\left(y_{t-1}, \tau\right) I\left[\left|y_{t-1}\right| \leq \tau\right]-\widehat{\alpha}(\tau) y_{t-1} I\left[\left|y_{t-1}\right|>\tau\right]\right)^{2}$, and $\widehat{h}_{\mathrm{cv}}$ is chosen such that

$$
\widehat{h}_{\mathrm{cv}}=\arg \min _{h \in H_{n}} \frac{1}{n} \sum_{t=1}^{n}\left(y_{t} I\left[\left|y_{t-1}\right| \leq \widehat{\tau}\right]-\widehat{g}_{-t}\left(y_{t-1} ; h\right) I\left[\left|y_{t-1}\right| \leq \widehat{\tau}\right]\right)^{2},
$$

with $\widehat{g}_{-t}\left(y_{t-1} ; h\right)=\frac{\sum_{s=1, \neq t}^{n} K\left(\frac{y_{t-1}-y_{s-1}}{h}\right) y_{s} I\left[\left|y_{s-1}\right| \leq \widehat{\tau}\right]}{\sum_{s=1, \neq t}^{n} K\left(\frac{y_{t-1}-y_{s-1}}{h}\right) I\left[\left|y_{s-1}\right| \leq \widehat{\tau}\right]}$ and $H_{n}=\left[n^{-1}, n^{-\left(1-\delta_{0}\right)}\right]$, in which $0<\delta_{0}<1$ is chosen such that $\widehat{h}_{\mathrm{cv}}$ is achievable and unique in each individual case.

We are interested in the following cases:

- Case A: $g(y)=\frac{1}{1+y^{2}}, \alpha=1$ and $\tau=2.5$; and

- Case B: $g(y)=y^{2}, \alpha=1$ and $\tau=2.5$.

Consider the cases of $n=250,600$ and 1000. Let $\widehat{g}_{j}(y)$ be the estimated function of $\widehat{g}(y)$ at the $j$-th replication and $y_{t}(j)$ be the generated value of $y_{t}$ at the $j$-th replication.

- Calculate the standard deviation and the bias of the form

$$
\operatorname{std}(\widetilde{\alpha})=\sqrt{\frac{1}{N-1} \sum_{j=1}^{N}(\widetilde{\alpha}(j)-\widetilde{\alpha})^{2}} \text { and } \operatorname{Bias}(\widetilde{\alpha})=\frac{1}{N} \sum_{j=1}^{N}(\widetilde{\alpha}(j)-\alpha)
$$

for Cases A and B separately under $N=1000$, where $\overline{\widetilde{\alpha}}=\frac{1}{N} \sum_{j=1}^{N} \widetilde{\alpha}(j)$.

- Calculate the Root Mean Squred Error of the form

$$
\operatorname{RMSE}(\widetilde{\alpha})=\sqrt{\frac{1}{N-1} \sum_{j=1}^{N}(\widetilde{\alpha}(j)-\alpha)^{2}}
$$

for Cases A and B separately under $N=1000$.

- For the case of $n=250,600$ and 1000, $N=1000$ and Cases A and B, calculate the average of the standard deviations of the form

$$
\operatorname{std}(\widehat{g})=\sqrt{\frac{1}{N} \frac{1}{n-1} \sum_{j=1}^{N} \sum_{t=2}^{n}\left(\widehat{g}_{j}\left(y_{t-1}(j)\right)-\overline{\hat{g}}(j)\right)^{2} I\left[\left|y_{t-1}(j)\right| \leq \widehat{\tau}\right]}
$$


where $\overline{\widehat{g}}(j)=\frac{1}{n-1} \sum_{t=2}^{n} \widehat{g}_{j}\left(y_{t-1}(j)\right)$.

- For the case of $n=250,600$ and 1000, $N=1000$ and Cases A and B, calculate the average of the bias of the form

$$
\operatorname{Bias}(\widehat{g})=\frac{1}{N} \frac{1}{n-1} \sum_{j=1}^{N} \sum_{t=2}^{n}\left(\widehat{g}_{j}\left(y_{t-1}(j)\right)-\overline{\widehat{g}}(j)\right) I\left[\left|y_{t-1}(j)\right| \leq \widehat{\tau}\right],
$$

where $\overline{\widehat{g}}(j)=\frac{1}{n-1} \sum_{t=2}^{n} \widehat{g}_{j}\left(y_{t-1}(j)\right)$.

- For the case of $n=250,600$ and 1000, $N=1000$ and Cases A and B, calculate the average of the Root Mean Squared Error of the form

$$
\operatorname{RMSE}(\widehat{g})=\sqrt{\frac{1}{N} \frac{1}{n-1} \sum_{j=1}^{N} \sum_{t=2}^{n}\left(\widehat{g}_{j}\left(y_{t-1}(j)\right)-g\left(y_{t-1}(j)\right)\right)^{2} I\left[\left|y_{t-1}(j)\right| \leq \widehat{\tau}\right]}
$$

Table 4.4 Simulation Results for Case A and Case B

\begin{tabular}{||c||c||c|c|c||c|c|c||}
\hline \multicolumn{1}{||c||}{} & \multicolumn{3}{c||}{ Case A } & \multicolumn{3}{c||}{ Case B } \\
\hline \multirow{5}{*}{ bias } & $n$ & $\widetilde{\alpha}$ & $\widehat{\tau}$ & $\widehat{g}$ & $\widetilde{\alpha}$ & $\widehat{\tau}$ & $\widehat{g}$ \\
\hline \multirow{3}{*}{ std } & 250 & -0.0381 & 0.3672 & 0.0438 & -0.0028 & -0.4244 & -0.0128 \\
& 1000 & -0.0103 & 0.3332 & 0.0446 & -0.0015 & -0.4321 & -0.0083 \\
& 250 & 0.0902 & 0.2198 & 0.4566 & 0.0058 & 0.2293 & 0.5865 \\
& 600 & 0.0185 & 0.1938 & 0.4516 & 0.0030 & 0.2291 & 0.4719 \\
& 1000 & 0.0116 & 0.1677 & 0.4514 & 0.0020 & 0.1997 & 0.4195 \\
\hline \multirow{3}{*}{ RMSE } & 250 & 0.0979 & 0.4236 & 0.3838 & 0.0079 & 0.5908 & 0.3706 \\
& 600 & 0.0211 & 0.4044 & 0.3540 & 0.0034 & 0.4893 & 0.2754 \\
& 1000 & 0.0131 & 0.3969 & 0.3294 & 0.0022 & 0.4875 & 0.2312 \\
\hline
\end{tabular}

Table 4.4 also shows that the rate of $\widetilde{\alpha}$ to $\alpha$ is much faster than that of $\widehat{g}$ to $g$ as shown in Theorem 3.1. Unlike Examples 4.1-4.3, the simulation study in Example 4.4 is more computationally intensive. This is because of the involvement of the nonparametric kernel estimation procedure and the cross-validation (CV) bandwidth 
selection method. Due to this, Table 4.4 provides only the simulation study results for the sample sizes of up to $n=1000$. Meanwhile, we have only used the CV selection method in practice. Theoretical discussion about such an issue requires further study and is therefore left for future research.

Example 4.5 Finally, as a real data illustration, we now look at the logarithm of the British pound/American dollar real exchange rate, $y_{t}$, defined as $\log \left(e_{t}\right)+\log \left(p_{t}^{U K}\right)-$ $\log \left(p_{t}^{U S A}\right)$, where $\left\{e_{t}\right\}$ is the monthly average of the nominal exchange rate, and $\left\{p_{t}^{i}\right\}$ denotes the consumption price index of country $i$.

These CPI data come from website: http://www.rateinflation.com/ and the exchange rate data are available at http://www.federalreserve.gov/, spanning from January 1988 to February 2011, with sample size $n=278$.

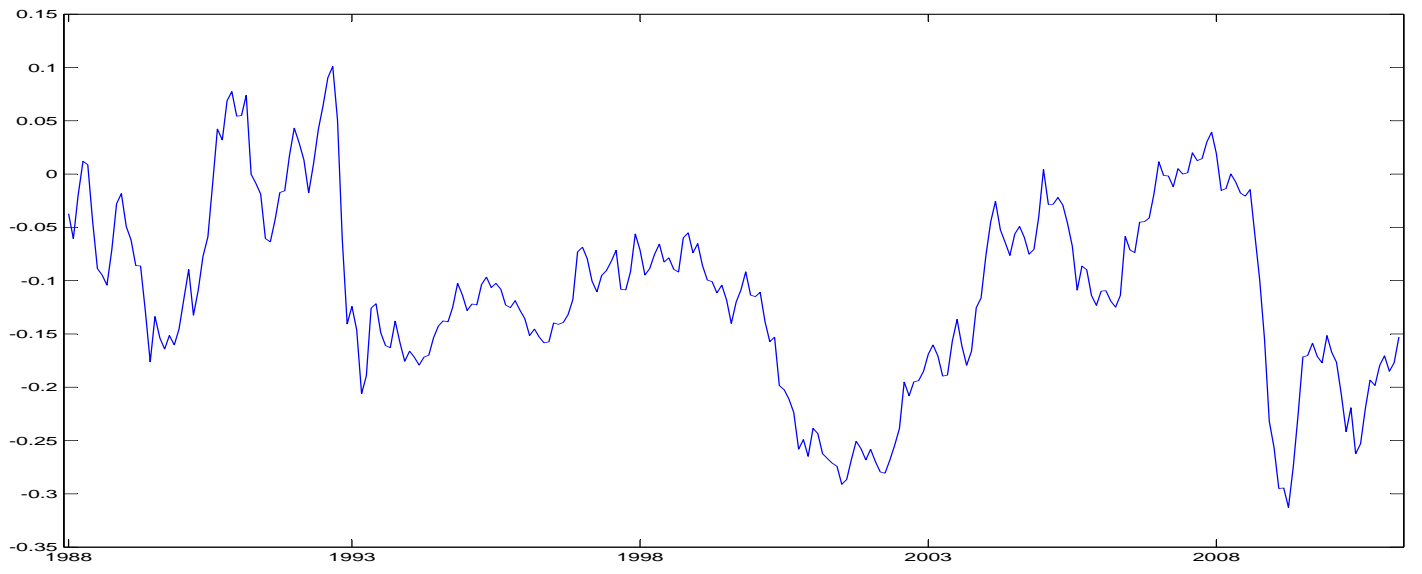

Figure 1: $y_{t}=\log \left(e_{t}\right)+\log \left(p_{t}^{U K}\right)-\log \left(p_{t}^{U S A}\right)$.

Our estimation method suggests the threshold models of the form $y_{t}$ as

$$
y_{t}=-0.1249 y_{t-1} I\left[\left|y_{t-1}\right| \leq 0.0134\right]+0.9974 y_{t-1} I\left[\left|y_{t-1}\right|>0.0134\right]+e_{t}
$$

and $\widehat{\sigma}^{2}=5.7808 \times 10^{-4}$. According to theory, we would expect that the number of observations in the stationary regime is proportional to $\sqrt{n}$, and in fact $\sum_{i=1}^{278} I\left[\left|y_{t-1}\right| \leq\right.$ $0.0134]=18$, which is small but the threshold effect is quite clear in the present example.

Actually, model (4.16) shows that while $\widetilde{\alpha}_{2}=0.9974$ is quite close to one, $\widetilde{\alpha}_{1}=$ 
-0.1249 is significantly different from one.

Model (4.16) implies

$$
y_{t}-y_{t-1}=-1.1249 y_{t-1} I\left[\left|y_{t-1}\right| \leq 0.0134\right]-0.0026 y_{t-1} I\left[\left|y_{t-1}\right|>0.0134\right]+e_{t}
$$

Thus, both models (4.16) and (4.17) indicate that $\left\{y_{t}\right\}$ may be nonstationary but does not necessary follow a random walk process. A similar discussion by Bec, Rahbek and Shephard (2008) also shows that the exchange rate between French franc and Deutsche mark over the time period between December 1972 and April 1988 does not necessarily follow a unit-root model. It is not easy to make a direct comparison since they use a shorter and different data set, and since they consider a Markov switching between regimes ultimately yielding a stationary model. This provides support from an empirical application point of view that there is some need to study a nonstationary threshold model of the form (1.1).

\section{Conclusions and discussion}

This paper has considered two classes of threshold autoregressive models with possible nonstationarity. The first one is a class of parametric threshold autoregressive (TAR) models with possible nonstationarity. The slope parameters have been consistently estimated. The second class is a new class of semiparametric threshold autoregressive (SEMI-TAR) models. We have estimated both the unknown slope parameter and unknown function using a semiparametrically consistent method.

One issue that has not been addressed is how to establish an asymptotic theory for $\widehat{\tau}$, a consistent estimate of $\tau$, in this kind of nonlinear and nonstationary situation. While it is anticipated that an asymptotically normal estimator of $\tau$ may be established (similar to Theorem 2 of Chan 1993), detailed assumptions and rigorous proofs may involve both new tools and more technicalities and therefore are left for future research.

Another issue is possible extensions of the current discussion for the first-order univariate case to higher-order and vector models. If the latter is achieved, one could introduce a class of threshold cointegration models with nonstationarity. Further discussion is also left for future research. Finally, there is the challenge of trying to extend 
the theory to smooth transition autoregressive (STAR) models. These models are extensively used in econometrics (see, for example, Terävirta, Tjøstheim and Granger 2010). Such an extension would be highly non-trivial and thus left for future research.

\section{Acknowledgments}

The authors would also like to acknowledge constructive suggestions and comments from seminar and conference participants at Monash University, Singapore Management University, University of Technology Sydney, and the University of Hong Kong. Special thanks go to Shiqing Ling for making their working paper available to us.

This work was started when the first author was visiting the University of Bergen in August/September in 2007. The work of the first author was supported financially by the University of Bergen and the Australian Research Council Discovery Grants under Grant Numbers: DP0558602 and DP1096374. The work of the third author was also supported financially by the Australian Research Council Discovery Grant under Grant Number: DP1096374.

\section{Appendix A}

In order to make this paper self-contained, we introduce some general results about $\beta$-null recurrent Markov chains in this appendix. At the request of a referee, we start by giving some econometric motivation for this class of processes.

One way to look at the $\beta$-null recurrent processes is that they can be thought of as a generalized I(1) class containing both linear and nonlinear models. (There are other generalizations as can be seen from Teräsvirta et al (2010, Section 11.4) or Tjøstheim (2011)). The starting point is the simple random walk. The two basic properties that Karlsen and Tjøstheim (2001) (KT) try to extend to a larger class of nonlinear I(1) type processes are (i) the persistence of the random walk (its nonstationarity); and (ii) the possibility of establishing central limit results.

The random walk is a linear process and a Markov chain. The Markov chain property also holds for the nonlinear generalization

$$
y_{t}=g\left(y_{t-1}\right)+\varepsilon_{t} \quad t \geq 1
$$


and such a process can be both stationary and nonstationary. If $|g(y)| \leq c|y|$ for some $c<1$ when $|y|$ is large enough, then there exists an initial distribution for $y_{0}$, so that $\left\{y_{t}\right\}$ becomes stationary if started with this distribution, and property (i) above is not fulfilled. On the other hand, if $g$ is such that $\left\{y_{t}\right\}$ is explosive, e.g. $g(y)=y^{2}$, then property (ii) cannot be satisfied in general; at least not in a nonparametric estimation context, because $\left\{y_{t}\right\}$ is then a transient Markov chain. A crucial property for $\left\{y_{t}\right\}$ to have for condition (ii) to hold is that it should be recurrent. This means that if $y_{s}=y$ for a certain time point $s$, then the Markov chain $\left\{y_{t}\right\}$ is guaranteed to be in an arbitrary small neighborhood around $y$ with probability one at a future time point; the process recurs or regenerates. We refer to KT for a more precise statement.

Under relatively weak regularity conditions, KT derive a central limit theorem for sums of the type $\sum_{s=1}^{n} h\left(y_{s}\right)$ properly scaled, where $h$ is a function satisfying some moment conditions. The key to this derivation is to use the recurrence property of the Markov chain to decompose the above sum as

$$
\sum_{s=1}^{n} h\left(y_{s}\right)=\sum_{s=1}^{\tau_{1}} h\left(y_{s}\right)+\sum_{s=\tau_{1}+1}^{\tau_{2}} h\left(y_{s}\right)+\ldots+\sum_{s=\tau_{T}+1}^{n} h\left(y_{s}\right)
$$

corresponding to the recurrence times $\tau_{1}, \tau_{2}, \ldots, \tau_{T} \leq n$; i.e., the time points of the regenerations of the chain. Clearly, $T=T(n) \rightarrow \infty$ as $n \rightarrow \infty$, but at a slower rate. Due to the Markov property, the components $\sum_{s=\tau_{i}+1}^{\tau_{i+1}} h\left(y_{s}\right), i=1, \ldots, T$ are independent and identically distributed, and this can be used to prove a central limit result under the additional assumption that the distribution of the recurrence time intervals $S_{i}=\tau_{i}-\tau_{i-1}$ should not have too heavy tails. More specifically, $\operatorname{Pr}\left\{S_{i}>s\right\}$ is essentially of the order $s^{-\beta}, 0<\beta<1$, so that $E\left[S_{i}^{k}\right]<\infty$ for $k<\beta$. This property is named $\beta$-null recurrence in KT. The random walk corresponds to $\beta=0.5$, as was established by Kallianpur and Robbins (1954).

Both parametric and nonparametric estimation can in principle be handled by this technique. A very different approach based on random walk-like processes and local time of the Wiener process has been used by Park and Phillips (2001) and Wang and Phillips (2009a, 2009b) but at present seems limited to a regression situation, although in other ways it is more general than the null recurrent Markov chain approach.

The class of recurrent Markov chains is subdivided into positive and null recurrent chains, depending on whether the expected recurrence time $E\left[S_{i}\right]$ is finite or not. The positive recurrent case has $E\left[S_{i}\right]<\infty(\beta=1$ in the above) and corresponds to stationarity, whereas the null recurrent case can be associated with a nonlinear extension of I(1). A unit root 
$\operatorname{AR}(p)$ process can be cast as a $p$-dimensional Markov chain, and in Myklebust, Karlsen and Tjøstheim (2011) it is shown that it is $\beta$-null recurrent with $\beta=0.5$ under weak assumptions. But the null recurrent class is not restricted to linear processes, and it has the useful invariance property that if $\left\{y_{t}\right\}$ is null recurrent ( $\beta$-null recurrent) then the transformed process $\left\{h\left(y_{t}\right)\right\}$ is null recurrent ( $\beta$-null recurrent) for an arbitrary one-to-one transformation $h$. Such an invariance property does not hold for the 'ordinary' I(1) class of processes. The class of $\beta$-null recurrent processes satisfies both (i) and (ii) above, but this set-up is restricted by the fact that it must be possible to embed $\left\{y_{t}\right\}$ in a Markov chain framework, and only one unit root is allowed. This is the case for model (1.1) that we are considering in this paper.

Let $\left\{y_{t}\right\}$ be a null recurrent Markov chain. We have $n$ observations of this process and replace the above sum of $h\left(y_{s}\right)$ by the following general form

$$
S_{n}(g)=\sum_{j=0}^{n} g\left(y_{j}, \ldots, y_{j+r-1}\right)
$$

for some function $g(\cdot)$. We let $\pi$ be an invariant measure for $\left\{y_{t}\right\}$ and

$$
S_{n}(g)=U_{0}+\sum_{k=1}^{T(n)} U_{k}+U_{(n)}
$$

be the decomposition of $S_{n}(g)$ as in (3.23) of KT. Moreover, $T(n)$ is the number of regenerations in the time interval $[0, n]$. We also need a notation for the moments w.r.t. the invariant measure $\pi$. Note from the decomposition (A.3) that

$$
U_{k}=U_{k}(g)=\sum_{\tau_{k-1}+1}^{\tau_{k}} g\left(y_{j}, \ldots, y_{j+r-1}\right), \quad k=1,2, \ldots,
$$

where the $\tau_{k}$-s are regeneration times. The $U_{k}^{\prime} s$ are identically distributed and are $(r-1)-$ dependent. (If $r=1$ they are independent). If they exist, we denote the expectation and variance of these terms by $\mu(g)=E\left(U_{k}(g)\right)$ and $\sigma^{2}(g)=\operatorname{var}\left(U_{k}(g)\right)$. Note that for $r=1$, $\mu(g)=\int g(x) \pi_{s}(d x)$ and similarly for $\sigma^{2}(g)$. For $r>1$

$$
\mu(g)=\pi_{s}(g) \equiv \int \pi_{s}\left(d x_{1}\right) P\left(x_{1}, d x_{2}\right) \cdots P\left(x_{r-1}, d x_{r}\right) g\left(x_{1}, \ldots, x_{r}\right)
$$

where $s$ refers to the small function used in the minimization condition (see (3.4) of KT 2001) and $P(\cdot, \cdot)$ is the transition probability of the chain. 
Finally, as in equation (4.4) of KT we introduce the notation

$$
\bar{\sigma}^{2}=\bar{\sigma}^{2}(g)=\sum_{k=-(r-1)}^{(r-1)} \operatorname{cov}\left(U_{1+|k|}(g), U_{1}(g)\right) .
$$

We are now ready to formulate the lemmas:

Assumption A.1. Assume that the minorization condition ((3.4) of KT) is fulfilled and that $\left\{y_{t}\right\}$ is $\beta$-null recurrent as defined in Definition 3.2 and in Theorem 3.1 of KT. We let $u(n)=n^{\beta} L_{s}(n)$ where $0<\beta<1$ and the slowly varying function $L_{s}(n)$ is as in the tail condition (3.16) of KT.

Lemma A.1 Let Assumption A.1 hold. (i) Let $\|g\| \in L_{r}^{1}\left(\pi_{s}\right)$ and also the process have an arbitrary initial distribution $\lambda$. Then as $n \rightarrow \infty$

$$
\frac{S_{n}(g)}{T(n)} \rightarrow \pi_{s}(g) \quad \text { almost surely (a.s.). }
$$

(ii) Then for $n$ large enough, the inequality $n^{\frac{1}{2}-\epsilon_{0}} \leq T(n) \leq n^{\frac{1}{2}+\epsilon_{0}}$ holds with probability one for some $0<\epsilon_{0}<\frac{1}{4}$.

Proof. The proof of (i) follows from that of Lemma 3.2 of KT (2001) while the proof of (ii) follows from Lemma 3.4 of KT (2001).

Lemma A.2 Let Assumption A.1 hold. If (i) $\mu(|g|)<\infty$ and (ii) there exists an $m>1$ so that $E|U(g)-\mu(g)|^{2 m} \leq d_{m}$ for some $d_{m}>0$, then

$$
\left(\Delta_{n}, T_{n}\right) \rightarrow_{D^{2}}\left(B\left(M_{\beta}\right), M_{\beta}\right) \text {, with } B \text { and } M_{\beta} \text { independent. }
$$

where the symbol " $\rightarrow D^{2}$ " means weak convergence in cadlag space (see, for example, the appendix of $K T$ 2001), $T_{n}=\left\{\frac{T([n t])}{u(n)}: t \geq 0\right\}, \Delta_{n}(t)=u^{-1 / 2}(n) \bar{\sigma}^{-1}(g)\left\{S_{[n t]}(g)-\mu(g) T([n t])\right\}$, $[n t]$ is the integer function and $M_{\beta}(t)$ is the Mittag-Leffler process as defined in KT on page 388.

Proof. The proof is essentially the same as the proof of Theorem $4.1 \mathrm{in} \mathrm{KT}$ but much simpler. As in that proof one introduces the scaled variables

$$
W_{k}(g)=\bar{\sigma}^{-1}\left(U_{k}(g)-\mu(g)\right) \text {. }
$$

(note that the existence of $\bar{\sigma}^{2}$ follows from condition ii), the definition of $\bar{\sigma}^{2}$ and the Schwartz 
inequality.) From condition ii) it also follows that there exists an $m>1$ such that $E\left[W_{k}^{2 m}\right]<$ $d_{m}^{\prime}$ for some constant $d_{m}^{\prime}$ from which

$$
n^{-m} \sum_{k=1}^{[n t]} E\left(W_{k}^{2 m}(g) \leq d_{m}^{\prime} t n^{-m-1}=o(1) .\right.
$$

It follows from standard limit theorems that

$$
Q_{n}(t) \doteq n^{-1 / 2} \sum_{k=1}^{[n t]} W_{k}(g) \rightarrow_{D} B(t) .
$$

Tightness is then proved exactly as in KT (note that there is a misprint in the last formula on page 393 of $\mathrm{KT}: W_{2 k-1}$ should be $\left.W_{2 k-i}\right)$. It follows that the convergence can be strengthened to convergence in $D^{2}$. We can neglect the edge terms

$$
\delta_{g, n}(t) \equiv u^{-1 / 2}(n) \bar{\sigma}^{-1}(g)\left\{U_{0}(t)+U_{(n)}(t)\right\}
$$

using the technique of part 2 of the proof of KT. The final part of the proof of KT only deals with the process $T_{n}$ induced by the number of regenerations $T(n)$, and this is completely independent of the bandwidth considerations introduced in KT. The lemma follows.

The limit distribution in Lemma A.2 is non-Gaussian. However, as in Theorem 4.2 of KT (2001), a Gaussian distribution can be obtained by a stochastic normalization. We let $T_{C}(n)$ denote the number of visits of $X_{t}$ to a small set $C$ in the time period $[0, n]$. We have that $T_{C}(n) / T(n)$ converges with probability 1 to $\pi_{s}(C)$. We now have the following lemma.

Lemma A.3 Let the conditions of Lemma A.2 hold and let $C$ be a small set. Then

$$
T_{C}^{1 / 2}(n) \pi_{s}^{1 / 2}(C) \bar{\sigma}^{-1}(g)\left\{T_{C}^{-1}(n) S_{n}(g)-\pi_{s}^{-1}(C) \mu(g)\right\} \stackrel{d}{\longrightarrow} N(0,1) .
$$

In Lemma A.2 the process $B\left(M_{\beta}(t)\right)$ enters. Concerning the existence of moments we have the following lemma.

Lemma A.4 Let $k$ be a positive integer. Then, $E\left[B\left(M_{\beta}(t)\right)^{2 k+1}\right]=0$, and

$$
E\left[B\left(M_{\beta}(t)\right)^{2 k}\right]=\frac{(2 k-1)(2 k-3) \cdots 1 \cdot t^{\beta k}}{(\Gamma(1+\beta))^{k}} .
$$

Proof. We use double expectation and the independence of the processes $B$ and $M_{\beta}$ to 
obtain

$$
\begin{aligned}
E\left[B\left(M_{\beta}(t)\right)^{2 k}\right] & =E\left[E\left(B\left(M_{\beta}(t)\right)^{2 k} \mid M_{\beta}(t)\right)\right]=E\left[(2 k-1)(2 k-3) \cdot 1 M_{\beta}(t)^{k}\right] \\
& =\frac{(2 k-1)(2 k-3) \cdots 1 \cdot t^{\beta k}}{(\Gamma(1+\beta))^{k}},
\end{aligned}
$$

so that all moments exist.

\section{Appendix B}

This appendix provides the detailed proofs of the lemmas and theorems stated in Sections 2 and 3 .

Proof of Lemma 2.1: Since the proof follows from that of Lemma 3.1 for the case of $g(y)=\alpha_{1} y$, we omit the detail here.

Proof of Lemma 2.3: Recall from Lemma 2.2 that as $n \rightarrow \infty$

$$
Q_{n}(r)=\frac{1}{\sqrt{n}} \sum_{t=1}^{[n r]} u_{t}+\frac{1}{\sqrt{n}} \sum_{t=1}^{[n r]} e_{t} \rightarrow_{D} \sigma B(r)+M_{\frac{1}{2}}(r) m_{u} \equiv Q(r)
$$

uniformly in $0<r \leq 1$.

We then start by proving (2.10) and (2.11). It follows from Lemma A.1(i) that as $n \rightarrow \infty$

$$
\frac{1}{T(n)} \sum_{t=1}^{n} y_{t-1}^{i} I\left[y_{t-1} \in C_{\tau}\right] \rightarrow_{P} \int_{-\infty}^{\infty} y^{i} I\left[y \in C_{\tau}\right] \pi_{s}(d y) \quad \text { for } i=1,2,
$$

which implies the proof of (2.10).

Let $b_{t}=y_{t-1} I\left[y_{t-1} \in C_{\tau}\right]$ and $S_{n 1}=\sum_{t=1}^{n} b_{t} e_{t}$. Since $\left\{e_{t}\right\}$ and $\left\{y_{s}\right\}$ are assumed to be independent for all $t>s$, Lemma 3.1 of Karlsen, Myklebust and Tjøstheim (2007) shows that $\left\{\left(y_{t-1}, e_{t}\right)\right\}$ is $\beta=\frac{1}{2}$-null recurrent. Using the fact that $\frac{T_{C}(n)}{\pi_{s}(C)} \frac{1}{T(n)}=1+o_{P}(1)$, Lemma A.3 implies that as $n \rightarrow \infty$

$$
\frac{1}{\sqrt{T(n)}} \sum_{t=1}^{n} y_{t-1} e_{t} I\left[y_{t-1} \in C_{\tau}\right] \stackrel{d}{\longrightarrow} N\left(0, \sigma_{1}^{2}\right)
$$

in which we have used (B.2) to derive $\frac{1}{T(n)} \sum_{t=1}^{n} b_{t}^{2} \rightarrow_{P} \int_{-\infty}^{\infty} y^{2} I\left[y \in C_{\tau}\right] \pi_{s}(d y)$. This completes the proof of (2.12).

We then prove equations (2.11) and (2.13). Recall that $y_{t}=\sum_{s=1}^{t} u_{s}+\sum_{s=1}^{t} e_{s}$. We now 
start to prove (2.11). Let $X_{n}(r)=\frac{1}{\sqrt{n}} \sum_{s=1}^{[n r]}\left(u_{s}+e_{s}\right)$. By the same arguments as in the proof of Theorem 3.1(a) of Phillips (1987), we have as $n \rightarrow \infty$

$$
\begin{aligned}
\frac{1}{n^{2}} \sum_{t=1}^{n} y_{t-1}^{2} I\left[y_{t-1} \in D_{\tau}\right] & =\frac{1}{n^{2}} \sum_{t=1}^{n} y_{t-1}^{2}-\frac{1}{n^{2}} \sum_{t=1}^{n} y_{t-1}^{2} I\left[y_{t-1} \in C_{\tau}\right] \\
& =\frac{1}{n^{2}} \sum_{t=1}^{n} y_{t-1}^{2}+o_{P}(1) \\
& =\int_{0}^{1} X_{n}^{2}(r) d r+o_{P}(1) \\
& \stackrel{d}{\longrightarrow} \int_{0}^{1} Q^{2}(u) d u
\end{aligned}
$$

where (B.4) follows from the fact that $\frac{1}{T(n)} \sum_{t=1}^{n} y_{t-1}^{2} I\left[y_{t-1} \in C_{\tau}\right] \rightarrow_{P} \mu_{2}$ by Lemmas 2.1 and A.1(i), and Lemma 2.2 has been used in (B.5). The proof of (2.11) is now completed.

Recall $\eta_{t}=u_{t}+e_{t}$. We finally prove (2.13). Note that

$$
\begin{aligned}
\frac{1}{n} \sum_{t=1}^{n} y_{t-1} e_{t} I\left[y_{t-1} \in D_{\tau}\right] & =\frac{1}{n} \sum_{t=1}^{n} y_{t-1} e_{t}-\frac{1}{n} \sum_{t=1}^{n} y_{t-1} e_{t} I\left[y_{t-1} \in C_{\tau}\right] \\
& =\frac{1}{n} \sum_{t=1}^{n} y_{t-1} e_{t}+o_{P}(1) \\
& =\frac{1}{n} \sum_{t=1}^{n} y_{t-1} \eta_{t}-\frac{1}{n} \sum_{t=1}^{n} y_{t-1} u_{t}+o_{P}(1) \\
& =\frac{1}{n} \sum_{t=1}^{n} y_{t-1} \eta_{t}-\frac{\left(\alpha_{1}-1\right)}{n} \sum_{t=1}^{n} y_{t-1}^{2} I\left[y_{t-1} \in C_{\tau}\right]+o_{P}(1) \\
& =\frac{1}{n} \sum_{t=1}^{n} y_{t-1} \eta_{t}+o_{P}(1) \\
& =\frac{1}{n} \sum_{t=2}^{n}\left(\sum_{s=1}^{t-1} \eta_{s}\right) \eta_{t}+o_{P}(1) \\
& =\frac{1}{2 n} \sum_{t=1}^{n}\left(\sum_{s=1}^{n} \eta_{s}\right) \eta_{t}-\frac{1}{2 n} \sum_{t=1}^{n} \eta_{t}^{2}+o_{P}(1) \\
& \stackrel{d}{\longrightarrow}\left(Q^{2}(1)-\sigma^{2}\right)
\end{aligned}
$$

where $Q(r)=\sigma B(r)+M_{\frac{1}{2}}(r) m_{u}$, equation (2.12) has been used in (B.6), Lemma A.1(i) has been used in (B.7) and Lemma 2.2 has been used in (B.8).

Therefore, the proof of Lemma 2.3 is completed. 
Proof of TheOREM 2.1: Recall that

$$
\begin{aligned}
\widehat{\alpha}_{1}-\alpha_{1} & =\frac{\sum_{t=1}^{n} e_{t} y_{t-1} I\left[y_{t-1} \in C_{\tau}\right]}{\sum_{t=1}^{n} y_{t-1}^{2} I\left[y_{t-1} \in C_{\tau}\right]} \text { and } \\
\widehat{\alpha}_{2}-1 & =\frac{\sum_{t=1}^{n} e_{t} y_{t-1} I\left[y_{t-1} \in D_{\tau}\right]}{\sum_{t=1}^{n} y_{t-1}^{2} I\left[y_{t-1} \in D_{\tau}\right]} .
\end{aligned}
$$

The proof of Theorem 2.1 then follows immediately from Lemma 2.3 and the continuous mapping theorem.

Proof of Lemma 3.1: We shall use Theorem 3.1 of KT to show that $\left\{y_{t}\right\}$ of (B.11) below is $\beta$-null recurrent with $\beta=\frac{1}{2}$ as in the random walk case. Recall the structure of model

$$
y_{t}=g\left(y_{t-1}\right) I\left[y_{t-1} \in C_{\tau}\right]+\alpha y_{t-1} I\left[y_{t-1} \in D_{\tau}\right]+e_{t},
$$

where $C_{\tau}$ is either a compact subset of $R^{1}$ or $C_{\tau}=(-\infty, \tau]$ or $C_{\tau}=[\tau, \infty)$ and $D_{\tau}$ is the complement of $C_{\tau}$.

Then the process $\left\{y_{t}\right\}$ is null recurrent (see Appendix B2 of Meyn and Tweedie 1994). Note that the proof in that book is easily modified to the situation of model (3.1) and a bounded $g(\cdot)$, see the remark at the bottom of page 303). This implies that there exists an invariant measure $\pi$ and that the process recurs with probability 1 , but with infinite expected recurrence time. The next step is to establish that the minorization condition (3.4) of KT holds. We first look at the case where $C_{\tau}$ is compact. Then the construction of Example 3.1 of KT can be used. The minorization condition then follows directly from Example 3.1 of KT with $f(x)$ of that paper given by $f(x)=g(x) I(x \in C)+x(1-I(x \in C))$ with $C=C_{\tau}$ since it is assumed that the distribution of $e_{t}$ is absolutely continuous with respect to Lebesgue measure. The fact that the minorization condition holds means that the split chain can be used, and as in KT, $\left\{S_{\alpha}\right\}$ is used to denote the recurrence times. They are iid and because of null recurrence $P\left(S_{\alpha}>n\right)$ must be asymptotically larger than $L_{s}(n) / n^{1+\varepsilon}$, where $L_{s}(n)$ is slowly varying and $\varepsilon>0$.

We are free to choose any small set $K_{0}$ as a set of regeneration in (B.11). We choose $K_{0}$ as $C_{\tau}$ if $C_{\tau}$ is compact. This is because compact sets are small if the distribution of $\left\{e_{t}\right\}$ is absolutely continuous with respect to Lebesgue measure. There are two ways in which $\left\{y_{t}\right\}$ may regenerate:

1. The process $\left\{y_{t}\right\}$ does not leave the set $C_{\tau}$ before it regenerates. Let $A_{n}$ be the event that $y_{t}$ stays in $C_{\tau}$ in at least $n+i$ steps and regenerates at step $n+i$ for $i \geq 1$. The time 
$S^{\prime}$ to regeneration satisfies

$$
P\left(S^{\prime}>n\right)=P\left(A_{n}\right) \leq \sum_{i=n+1}^{\infty} \rho^{i} \leq M \rho^{n+1}=o\left(n^{-\gamma}\right)
$$

for any $0<\gamma<1$, where $0<M<\infty$ is an absolute constant. Here $\rho=\rho_{1} \rho_{2}$ where $\rho_{1}=\sup _{x \in C_{\tau}} P\left(x, C_{\tau}\right)$, where $P(\cdot, \cdot)$ is the transition probability of the chain. Note that $0<\rho_{1}<1$. Similarly, $\rho_{2}=1-a$, where $a$ is defined in Example 3.1 of KT and $0<a<1$. From this, comparing to $O\left(n^{-\gamma}\right)$, it is seen that these recurrence times do not contribute to the tail bahaviour of $S_{\alpha}$.

2. The process $\left\{y_{t}\right\}$ does leave the set $C_{\tau}$ before it regenerates. Outside the set $C_{\tau},\left\{y_{t}\right\}$ behaves as a random walk, and therefore according to the paper by Kallianpur and Robbins (1954) and the fact that what goes on inside the set $C_{\tau}$ can be neglected compared to a probability of order $O\left(n^{-1 / 2}\right)$, if $S^{\prime \prime}$ is such a recurrence time, $P\left(S^{\prime \prime}>n\right)=O\left(n^{-1 / 2}\right)$. This means that the tail behaviour of $S_{\alpha}$ is controlled by the tail behaviour of $S^{\prime \prime}$ and that $\left\{y_{t}\right\}$ is $\beta$-null recurrent with $\beta=\frac{1}{2}$.

Next we look at the case where $C_{\tau}=(-\infty, \tau]$ or $[\tau, \infty)$. Without loss of generality, we may assume $C_{\tau}=(-\infty, \tau]$ In this case we let the set of regeneration be the set $K_{0}=\left[\tau^{\prime}, \tau\right]$ where $\tau^{\prime}$ can be taken to be any real number smaller than $\tau$. From Assumption 3.1(iv), we may assume that $\left\{y_{t}\right\}$ behaves as a stationary process to the left of $\tau^{\prime}$ and like a random walk to the right of $\tau$.

Again it follows from Appendix B2 of Meyn and Tweedie (1994) that $\left\{y_{t}\right\}$ is null recurrent. (In fact Meyn and Tweedie has $g(\cdot)$ linear). By the same reasoning as above, option 2 then splits into two cases: $2 \mathrm{a})$ where $\left\{y_{t}\right\}$ leaves $K_{0}$ going to the stationary part of $\left\{y_{t}\right\}$ and then does not enter the random walk part before it regenerates. The associated recurrence time $S^{\prime \prime \prime}$ has tail behaviour controlled by $P\left(S^{\prime \prime \prime}>n\right)=O\left(L_{s}(n) / n^{1+\varepsilon}\right)$. The possibility $\left.2 \mathrm{~b}\right)$ is the case where the random walk part is visited before it regenerates, but here $P\left(S^{\prime \prime}>n\right)=O\left(n^{-1 / 2}\right)$, as time spent in the stationary part and in the set $C_{\tau}$ can be neglected as far as tail behaviour is concerned. This implies again that $\left\{y_{t}\right\}$ is $\beta$-null recurrent with $\beta=\frac{1}{2}$.

REMARK: The process $\left\{y_{t}\right\}$ may even be explosive on the left-hand side, if it explodes in the direction of $K_{0}$ and the random walk regime. This is illustrated by the simulated example in Example 4.3.

Proof of Theorem 3.1: Because of Lemma 3.1, the proof of (3.8) is the same as that 
of (2.15). Let $W_{n t}(y)=\frac{K\left(\frac{y-y_{t-1}}{h}\right) I\left[y_{t-1} \in C_{\tau}\right]}{\sum_{t=1}^{n} K\left(\frac{y-y_{t-1}}{h}\right) I\left[y_{t-1} \in C_{\tau}\right]}$. In order to prove (3.7), in view of (3.6), it suffices to show that as $n \rightarrow \infty$

$$
\begin{aligned}
& \sqrt{\sum_{t=1}^{n} K\left(\frac{y-y_{t-1}}{h}\right) I\left[y_{t-1} \in C_{\tau}\right]} \sum_{t=1}^{n} W_{n t}(y)\left(g\left(y_{t-1}\right)-g(y)\right) \rightarrow_{P} 0, \\
& \sqrt{\sum_{t=1}^{n} K\left(\frac{y-y_{t-1}}{h}\right) I\left[y_{t-1} \in C_{\tau}\right]} \sum_{t=1}^{n} W_{n t}(y) e_{t} \stackrel{d}{\longrightarrow} N\left(0, \sigma^{2}\right) .
\end{aligned}
$$

Note that by Taylor expansions, Lemma A.1(i) and Assumption 3.1

$$
\begin{aligned}
& \frac{1}{T(n) h} \sum_{t=1}^{n} K\left(\frac{y-y_{t-1}}{h}\right) I\left[y_{t-1} \in C_{\tau}\right]=I\left[y \in C_{\tau}\right] p_{s}(y)\left(1+o_{P}(1)\right), \\
& \frac{1}{T(n) h} \sum_{t=1}^{n} K\left(\frac{y-y_{t-1}}{h}\right) I\left[y_{t-1} \in C_{\tau}\right]\left(g\left(y_{t-1}\right)-g(y)\right) \\
& =\frac{g^{\prime}(y) h}{T(n) h} \sum_{t=1}^{n} K\left(\frac{y-y_{t-1}}{h}\right) I\left[y_{t-1} \in C_{\tau}\right] \frac{\left(y_{t-1}-y\right)}{h} \\
& +\frac{g^{\prime \prime}(u) h^{2}}{2 T(n) h} \sum_{t=1}^{n} K\left(\frac{y-y_{t-1}}{h}\right) I\left[y_{t-1} \in C_{\tau}\right] \frac{\left(y_{t-1}-y\right)^{2}}{h^{2}} \\
& =g^{\prime}(y) h \int v K(v) d v I\left[y \in C_{\tau}\right] p_{s}(y)+o_{P}(h) \\
& +\frac{h^{2} g^{\prime \prime}(y)}{2} \int v^{2} K(v) d v I\left[y \in C_{\tau}\right] p_{s}(y)+o_{P}\left(h^{2}\right) \\
& =o_{P}(h)
\end{aligned}
$$

where $u$ is between $y$ and $y_{t-1}$, and $p_{s}(y)$ is the density function of the invariant measure $\pi_{s}$ of $\left\{y_{t}\right\}$. This, along with Lemma A.1(ii) and using Assumption 3.1(iii), implies (B.12).

Let $a_{h t}(y)=K\left(\frac{y-y_{t-1}}{h}\right) I\left[y_{t-1} \in C_{\tau}\right]$ and $S_{n 2}=\sum_{t=1}^{n} a_{h t}(y) e_{t}$. Since $\left\{e_{t}\right\}$ and $\left\{y_{s}\right\}$ are assumed to be independent for all $t>s$, Lemma 3.1 of Karlsen, Myklebust and Tjøstheim (2007) shows that $\left\{\left(y_{t-1}, e_{t}\right)\right\}$ is $\beta=\frac{1}{2}$-null recurrent. Using the fact that $\frac{T_{C}(n)}{\pi_{s}(C)} \frac{1}{T(n)}=$ $1+o_{P}(1)$, Lemma A.3 and the proof of Theorem 5.4 of KT imply that as $n \rightarrow \infty$

$$
\begin{aligned}
& \frac{\sqrt{\sum_{t=1}^{n} K^{2}\left(\frac{y-y_{t-1}}{h}\right) I\left[y_{t-1} \in C_{\tau}\right]}}{\sqrt{\sum_{t=1}^{n} K\left(\frac{y-y_{t-1}}{h}\right) I\left[y_{t-1} \in C_{\tau}\right]}} \cdot \frac{\sum_{t=1}^{n} K\left(\frac{y-y_{t-1}}{h}\right) I\left[y_{t-1} \in C_{\tau}\right] e_{t}}{\sqrt{\sum_{t=1}^{n} K^{2}\left(\frac{y-y_{t-1}}{h}\right) I\left[y_{t-1} \in C_{\tau}\right]}} \\
& \stackrel{d}{\longrightarrow} N\left(0, \sigma^{2} \int_{-\infty}^{\infty} K^{2}(u) d u\right)
\end{aligned}
$$


in which we have followed (B.14) to derive

$$
\frac{1}{T(n) h} \sum_{t=1}^{n} K^{i}\left(\frac{y-y_{t-1}}{h}\right) I\left[y_{t-1} \in C_{\tau}\right] \rightarrow_{P} \int_{-\infty}^{\infty} K^{i}(u) d u \cdot I\left[y \in C_{\tau}\right] p_{s}(y)
$$

for $i=1,2$. This completes the proof of (B.13). The proof of Theorem 3.1 is therefore completed.

\section{REFERENCES}

Bec, F., Rahbek, A., Shephard, N., 1998. The ACR model: a multivariate dynamic mixture autoregression. Oxford Bulletin of Economics and Statistics 70, 583-618.

Billingsley, P., 1968. Convergence of Probability Measures. John Wiley, New York.

Cai, Z., Li, Q., Park, J., 2009. Functional-coefficient models for nonstationary time series data. Journal of Econometrics 148, 101-113.

Caner, M., Hansen, B. E., 2001. Threshold autoregression with a unit root. Econometrica 69, $1555-1596$.

Chan, K. S., 1990. Testing for threshold autoregression. Annals of Statistics 18, 1886-1894.

Chan, K. S., 1993. Consistency and limiting distribution of the least squares estimator of a threshold autoregressive model. Annals of Statistics 21, 520-533.

Chan, K. S., Tsay, R. S., 1998. Limiting properties of the least squares estimator of a continuous threshold autoregressive model. Biometrika 85, 413-426.

Chen, J., Gao, J., Li, D., 2008. Semiparametric regression estimation in null recurrent time series. Forthcoming in Bernoulli.

Fan, J., Yao, Q., 2003. Nonlinear Time Series: Nonparametric and Parametric Methods. Springer, New York.

Gao, J., 2007. Nonlinear Time Series: Semiparametric and Nonparametric Methods. Chapman \& Hall/CRC, London.

Gao, J., King, M. L., Lu, Z., Tjøstheim, D., 2009a. Specification testing in nonstationary time series autoregression. Annals of Statistics 37, 3893-3928.

Gao, J., King, M. L., Lu, Z., Tjøstheim, D., 2009b. Nonparametric specification testing for nonlinear time series with nonstationarity. Econometric Theory 25, 1869-1892.

Hall, P., Heyde, C. C., 1980. Martingale Limit Theory and Its Application. Academic Press, New York. 
Hansen, B. E., 1996. Inference when a nuisance parameter is not identified under the null hypothesis. Econometrica 64, 413-430.

Hansen, B. E., 2000. Sample splitting and threshold estimation. Econometrica 68, 575-603.

Kallianpur, G., Robbins, H., 1954. The sequence of sums of independent random variables. Duke Mathematical Journal 21, 285-307.

Karlsen, H. A., Tjøstheim, D.,2001. Nonparametric estimation in null recurrent time series. Annals of Statistics 29, 372-416.

Karlsen, H. A., Mykelbust, T., Tjøstheim, D., 2007. Nonparametric estimation in a nonlinear cointegration type model. Annals of Statistics 35, 252-299.

Li, D., Ling, S., 2011. On the least squares estimation of multiple-regime threshold autoregressive models. Forthcoming in Bernoulli.

Liu, W., Ling, S., Shao, Q., 2009. On nonstationary threshold autoregressive models. Working paper available from the second author at http://www.math.ust.hk/ ${ }^{\sim}$ maling.

Meyn, S. P., Tweedie, R. L., 1994. Markov Chains and Nonnegative Operators. Cambridge University Press.

Myklebust, T., Karlsen, H. A., Tjøstheim, D., 2011. Null recurrent unit root processes. Forthcoming in Econometric Theory.

Park, J., Phillips, P. C. B., 2001. Nonlinear regressions with integrated time series. Econometrica $69,117-162$.

Pham, D. T., Chan, K. S., Tong, H.. 1991. Strong consistency of the least squares estimator for a non-ergodic threshold autoregressive model. Statistica Sinica 1, 361-369.

Phillips, P. C. B., 1987. Time series regression with a unit root. Econometrica 55, 277-301.

Phillips, P. C. B., Park, J., 1998. Nonstationary density estimation and kernel autoregression. Cowles Foundation Discussion Paper, No. 1181, Yale University.

Teräsvirta, T., Tjøstheim, D., Granger, C. W. J., 2010. Modelling Nonlinear Economic Time Series. Oxford University Press, Oxford.

Tjøstheim, D., 2011. Modelling nonlinear and nonstationary time series. Forthcoming in Handbook in Time Series.

Tong, H., 1983. Threshold Models in nonlinear Time Series Analysis. Lecture Notes in Statistics 21. Springer-Verlag, New York

Tong, H., 1990. Nonlinear Time Series. A Dynamical System Approach. Oxford University Press, New York. 
Tong, H., Lim, K. S., 1980. Threshold autoregression, limit cycles and cyclical data (with Discussion). Journal of the Royal Statistical Society Series B 42, 245-292.

Wang, Q. Y., Phillips, P. C. B., 2009a. Asymptotic theory for local time density estimation and nonparametric cointegrating regression. Econometric Theory 25, 710-738.

Wang, Q. Y., Phillips, P. C. B., 2009b. Structural nonparametric cointegrating regression. Econometrica $77,1901-1948$. 